Reconstruction of road defects and road roughness classification using Artificial Neural Networks simulation and vehicle dynamic responses: Application to experimental data

Highlights

- Estimation of road profiles and classes using neural networks on measured data.

- Discrete obstacles are reconstructed with higher correlation than Belgian pave.

- Ride comfort mode has better quality in reconstructed profiles than handling mode.

- Consistently good approximations of DSDs occur between 0.2 and 1.8 cycles $/ \mathrm{m}$. 


\title{
Reconstruction of road defects and road roughness classification using Artificial Neural Networks simulation and vehicle dynamic responses: Application to experimental data
}

H.M. Ngwangwa ${ }^{a}$, P.S. Heyns ${ }^{a}$, H.G.A. Breytenbach ${ }^{\text {b }}$ and P.S. Els ${ }^{b}$

a. Dynamic Systems Group,

b. Vehicle Dynamics Group,

Department of Mechanical and Aeronautical Engineering, University of Pretoria, 0002 Pretoria, South Africa.

Tel: +2712 420 2432; Fax: +27123625087

E-mail: hngwangwa@gmail.com.

\begin{abstract}
This paper reports the performance of an Artificial Neural Network based road condition monitoring methodology on measured data obtained from a Land Rover Defender 110 which was driven over discrete obstacles and Belgian paving. In a previous study it was demonstrated, using data calculated from a numerical model, that the neural network was able to reconstruct road profiles and their associated defects within good levels of fitting accuracy and correlation. A nonlinear autoregressive network with exogenous inputs was trained in a series-parallel framework. When compared to the parallel framework, the series-parallel framework offered the advantage of fast training but had a shortcoming in that it required feed-forward of true road profiles. In this study, the true profiles are not available and the test data are obtained from field measurements. Training data are numerically generated by making minor adjustments to the real measured profiles and applying them to a full vehicle
\end{abstract}


model of the Land Rover. This is done to avoid using the same road profile and acceleration data for training and testing or validating the neural network. A static feedforward neural network is trained and consequently tested on the real measured data. The results show very good correlations over both the discrete obstacles and the Belgian paving. The random nature of the Belgian paving necessitated correlations to be made using their displacement spectral densities as well as evaluations of RMS error percent values of the raw road profiles. The use of displacement spectral densities is considered to be of much more practical value than the road profiles since they can easily be interpreted into road roughness measures by plotting them over an internationally recognized standard roughness scale.

Keywords: Road condition monitoring, Artificial neural networks, Vehicle model, Ride comfort, Handling, Road roughness assessment, Four state semi-active suspension, Road profile reconstruction, Displacement spectral density

\section{Introduction}

This work is a continuation of an ongoing study on road condition monitoring undertaken by the Dynamic Systems Group at the University of Pretoria. The seminal work was done by Hugo, Heyns, Thompson and Visser [1] on mine haul roads. Hugo et al. [1] employed an inverse tire model of the haul truck to calculate road profiles which would provide input into the development of a real time road maintenance management system for mine haul roads. As pointed out by Ngwangwa, Heyns, Labuschagne and Kululanga [2], the methodology's reliance on extensive system characterization limits its practical feasibility. Ngwangwa et al. [2] therefore proposed a neural network-based methodology of reconstructing the road profiles. 
The advantages of such a methodology cannot be overemphasised. Hugo et al. [1] discuss the need for development of optimal maintenance strategies. The existing maintenance techniques fail to optimize the cost function and they have potential to generate excessive costs due to over- or under-maintenance [1]. Too little maintenance leads to excessive vehicle operation and maintenance costs while excessive road maintenance generates extremely high costs that may not be justified against its benefit in reducing vehicle operation and vehicle maintenance costs.

Heyns, De Villiers and Heyns, [3] further note that the existing methods are timeconsuming and subjective with the effect that they may not be well-suited for use on road networks such as mine haul roads. Existing methodologies require too much management input through their need for costly regular inspections and subjective assessments of road segments and rather strict adherence to fixed schedules. The present methodology however, has the potential for development of a real-time maintenance management system which may require minimal subjective assessment. There is no need for stopping operations and data can be captured while operating.

In the previous paper [2], an eight degree-of-freedom (8-DOF) linear pitch-plane model with four degrees of freedom representing vehicle motions and additional four degrees for seat and driver vibrations, was employed for the investigation. Eight road roughness classes having well-known displacement spectral densities (DSDs) on the International Organization for Standardization power spectral density (ISO PSD) classification of road roughness [4] were applied to the model to calculate corresponding sprung mass and axle accelerations. The accelerations, serving as inputs, and the corresponding road profiles, serving as targets, were applied to a Non-linear Auto-Regressive with eXogenous inputs (NARX) network having 20 hidden neurons and one output neuron $[2]$. 
The vehicle model was subsequently simulated with different sets of road profiles which were generated by a random road surface function to calculate corresponding sprung mass and axle accelerations. The accelerations were accordingly applied to the trained NARX network to obtain the road profiles. The simulated road profiles were compared with the actual road profiles. These evaluations were performed for different road roughness conditions (based on the ISO PSD roughness scale [4]), different vehicle speeds, growing road defects, noisy conditions, and different vehicle payload conditions [2]. It was noted that the methodology was able to reconstruct the road profiles to within an error margin of $20 \%$ with a minimum correlation of $94 \%$ [2].

In this paper, the methodology is applied to field test data using an experimental Land Rover Defender 110. This vehicle has been extensively used by the Vehicle Dynamics Group at University of Pretoria and has a great deal of instrumentation permanently mounted on it [5]. It has been adapted for suspension tests to allow for suspension settings to be safely switched between ride comfort and handling modes [5], a system that is known as four state semi-active suspension $\left(\mathbf{4} \boldsymbol{S}_{4}\right)[4]$. The vehicle response measurements used in this paper were carried out by Breytenbach [6, 7] using an eDAQ lite measurement system while the road profiles were measured by Becker [8, 9] using a 'can-can' machine. The tests were performed at Gerotek, a world class vehicle testing infrastructure in Pretoria, South Africa. The tests were conducted over discrete obstacles and Belgian paving with the driving conditions changing between different combinations of two speeds and suspension settings from one test run to another. These would typically yield four possible test scenarios, namely, ride comfort mode at low speed, ride comfort mode at high speed, handling mode at low speed and handling mode at high speed though some of these combinations were not performed over the discrete obstacles. 
One of the most crucial issues with the use of neural networks in non-linear regression or function approximation is its generalization capabilities. The data used in this study was obtained to validate a mathematical model of the Land Rover and since most evaluations of vehicle vertical dynamics are generally conducted over discrete obstacles and random road surfaces [6], there was no need, in that study, for more road profile data other than the discrete obstacles and Belgian paving. However, in this study, such scarcity in the measured profile data poses a challenge as far as ensuring network generalization is concerned. This has been addressed in this paper by generating slightly altered versions of the actual measured road profiles which are applied to a full-vehicle seven degree-of-freedom model (7-DOF) of the Land Rover as formulated by Breytenbach $[6,7]$. The numerically generated data is used for training the neural network while the measured data is used for validating and testing the neural network.

The following section presents the properties of the Land Rover, its suspension forces and the numerical model which is used in generating the accelerations that serve as inputs when training the neural network.

\section{Land Rover properties, suspension and numerical model}

\subsection{Geometry and inertial properties}

The Land Rover geometry and inertial properties used in the study were characterized by Uys, Els, Thoresson, Voigt, and Combrinck [10] in a laboratory environment. The determination of the mass moments of inertia and the location of the vehicle's centre of gravity were of particular interest and were carried out by using the rotational vibration of a rigid body about a pivoting point, with a restoring force provided by a spring. Uys et al. [10] developed an ADAMS model of the Land Rover based on these properties which was later validated with field results by Els [5]. The details of these 
measurements and the procedures that were employed can be found in reference [10].

Table 1 summarises these vehicle inertial properties and geometry.

Table 1 Summary of vehicle inertial properties and geometry.

\section{Land Rover Property and Dimensions Quantity}

Sprung mass

$1734 \mathrm{~kg}$

Sprung mass pitch moment of inertia

$2440 \mathrm{~kg} \cdot \mathrm{m}^{2}$

Sprung mass roll moment of inertia

688 kg.m ${ }^{2}$

Front unsprung mass

$229 \mathrm{~kg}$

Front unsprung mass roll moment of

$33.1 \mathrm{~kg} . \mathrm{m}^{2}$

inertia

Rear unsprung mass

$229 \mathrm{~kg}$

Rear unsprung mass roll moment of inertia

$33.1 \mathrm{~kg} \cdot \mathrm{m}^{2}$

Centre of mass longitudinal position from

$1.395 \mathrm{~m}$

front tire centre

Centre of mass height

$1.19 \mathrm{~m}$

Wheel track width

$1.5 \mathrm{~m}$

Suspension track width

$1.1 \mathrm{~m}$

\subsection{The tire}

The tire is the most important component of the vehicle model as it acts as the interface between the vehicle and the road $[1,6,11]$. Ideally it must maintain traction on the road as it absorbs the road irregularities and because of the range of functions the tire must perform, it is also equally difficult to model. In most vehicle simulations, the tire imposes the largest limitation to achieving correlation with measured results [1]. In this 
study the tire is modelled as a point follower with a parallel linear spring and damper of $250000 \mathrm{~N} / \mathrm{m}$ and $1200 \mathrm{Ns} / \mathrm{m}$ respectively [6].

\subsection{The suspension forces}

Owing to its complex fluid dynamics and need to optimize vehicle performance between ride comfort and handling modes, the suspension dynamics of the $4 \mathrm{~S}_{4}$ could be oversimplified if it is assumed that suspension forces are directly proportional to suspension displacements and velocities $[5,6]$. There are several mathematical models proposed in the literature for complex damper behaviour but this study adopts the approach employed by Breytenbach $[6,7]$ where empirical models are employed. The suspension forces are considered to comprise five main contributing components namely: hydro-pneumatic spring forces, hydraulic damping force, friction force in the $\mathbf{4} \boldsymbol{S}_{4}$ suspension struts, bushing force in the vehicle trailing arms and bump stop forces [6]. The Land Rover's hydro-pneumatic spring is modelled as a polytropic gas compression process with constant area in eq. (1). [6]

$$
F_{s}=p_{s t a t} A\left(\frac{x_{s t a t}}{x}\right)^{n_{p}}
$$

where $F_{s}$ is the force in the hydro-pneumatic spring; $p_{\text {stat }}$ is static pressure $(2.167$ $\mathrm{MPa}) ; A$ is the bore cross-section area $\left(1.963 \times 10^{-3} \mathrm{~m}^{2}\right.$ equivalent to $50 \mathrm{~mm}$ diameter bore); $x_{\text {stat }}$ is the static displacement $(0.0509 \mathrm{~m}$ for handling mode and $0.255 \mathrm{~m}$ for ride comfort mode); $x$ is the hydro-pneumatic spring displacement; and $n_{p}$ is the polytropic gas constant equal to unity. The polytropic constant of unity represents an isothermal process and it was found that it adequately predicted the spring force in most tests [6]. The other four forces in the $\mathbf{4} \mathbf{S}_{\mathbf{4}}$ suspension are nonlinear and could not be modelled as linear functions as is often assumed. They were modelled empirically using MATLAB's 
interpolation function ppval.m [12]. Figure 1 shows the behaviour of these forces over some typical operating suspension displacements and velocities.
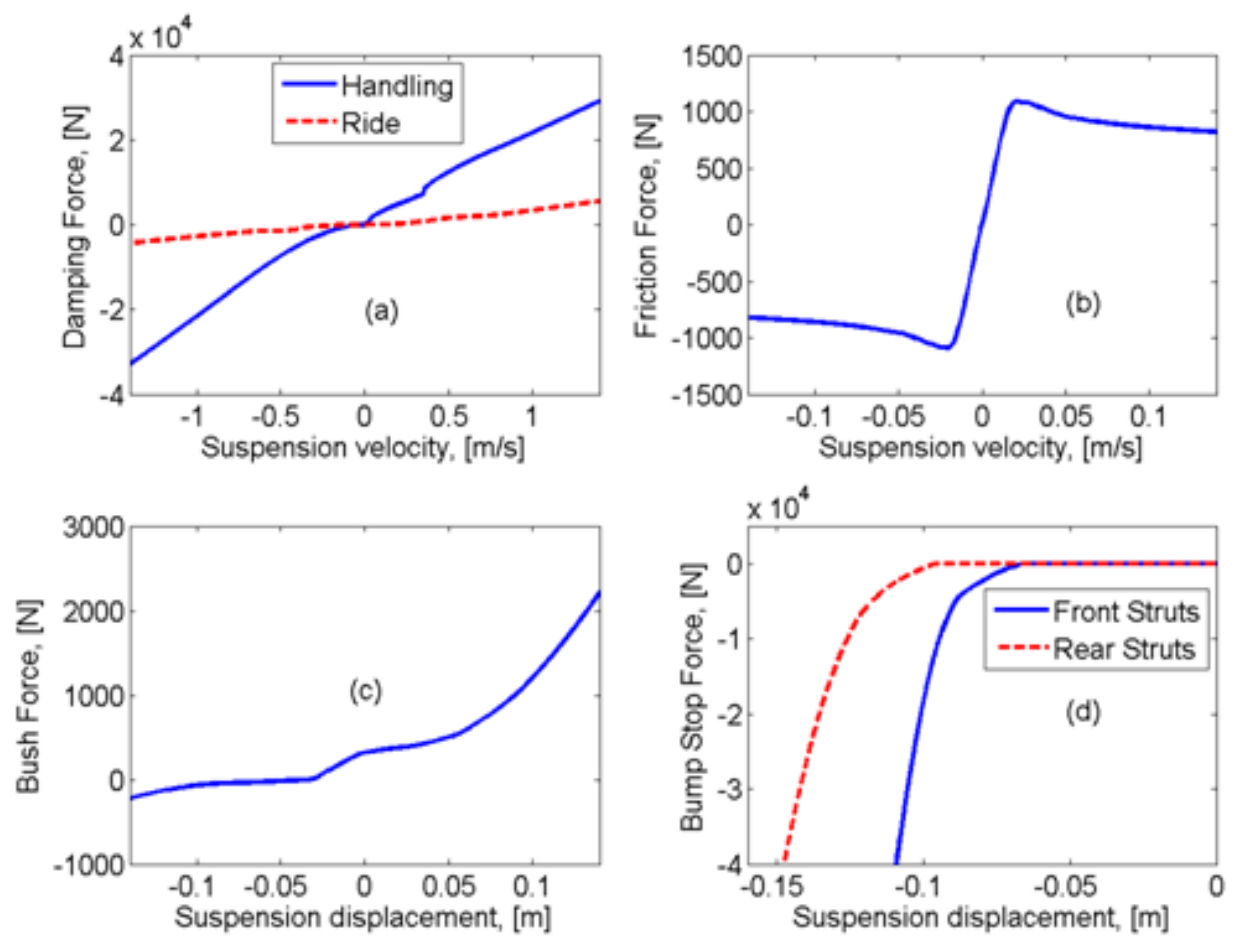

Figure 1. The $4 \mathrm{~S}_{4}$ suspension forces: (a) hydraulic damping forces for ride comfort (dashed) and handling mode (solid) (b) Friction forces (c) Bushing forces and (d) Bump stop forces for front struts (solid) and for rear struts (dashed).

Figure 1 (a) shows larger damping forces in the handling mode than in the ride comfort mode at all suspension velocities. This behaviour is uniform in all the suspension struts, though in practice, small variations within manufacturing tolerances have led to varying characteristics [6]. Figure 1 (b) shows that friction forces within the $\mathbf{4} \boldsymbol{S}_{\mathbf{4}}$ suspension are lower than the damping forces but its limiting static value is higher than the dynamic friction forces. The value of $0.02 \mathrm{~m} / \mathrm{s}$ is ideally too large for stick-slip velocity. This is caused by the participation of viscous damping effects on damper blocks in the valve assembly of the laboratory suspension strut. This fact was verified by another 
laboratory suspension strut which had no damper blocks in the valve assembly and it exhibited near-zero threshold velocity in the stick-slip transition region [6]. Since the suspension strut on the actual Land Rover under test has damper blocks, the former friction characteristic was utilized. Stribeck curves were consequently fitted to the measured ride and handling friction curves and averaged over the velocity range \pm 0.05 $\mathrm{m} / \mathrm{s}[6,7]$. Thus Figure 1(b) represents a generalised friction curve which is a result of averaging ride and handling friction curves. However during the actual simulations, specific ride and handling friction curves were employed.

The bushing forces in the trailing arms are also much lower than the damping forces for suspension displacements between $-0.1 \mathrm{~m}$ and $+0.05 \mathrm{~m}$, yet increase almost exponentially outside these limits (Figure 1 (c)). By design, the rear suspension struts have greater working space than front struts as shown in Figure $1(\mathrm{~d})$.

\subsection{The Vehicle Model}

The development of the full-car model of the Land Rover Defender 110 and validation against measured data was carried out by Breytenbach [6]. The model comprises translational motion in the vertical direction and rotational motions in the roll and pitch directions for the sprung mass as well as translational motion in the vertical direction and rotational motion in the roll direction for each of the unsprung masses (front and rear solid axles).

The full development of the model can be found in reference [6]. In this paper, only the seven equations of motion for each degree of freedom are presented and briefly discussed for clarity. 
The equation of motion for the sprung mass can be represented by eq.2.

$$
m_{b} \ddot{z}_{b}=m_{b} g-F_{s r r}-F_{s l r}-F_{s l f}-F_{s r f}
$$

where $m_{b}$ is the vehicle body mass, $\ddot{z}_{b}$ is the vertical body acceleration measured at its centre of gravity and $F_{s r r}, F_{s l r}, F_{s i f}$ and $F_{s l f}$ are the suspension forces measured on the struts at the right rear, left rear, right front and left front positions. Force of gravity on the sprung mass $m_{b} g$ is included to model sprung mass's free body motion in the vertical direction since occasionally the tires lose contact with the ground.

The forces in the suspension struts were modelled empirically using MATLAB's interpolation function ppval.m [12]. This function constructs a polynomial that fits the best line to the experimental data. The total suspension force in each strut is therefore determined by adding the spring forces, damper forces, friction forces, bump stop forces, and bushing forces. The force equation at the front axle is given by eq. 3

$$
\begin{aligned}
& m_{u f} \ddot{z}_{u f}+k_{t r f} z_{u f}+k_{t r f} c \theta+c_{t r f} \dot{z}_{u f}+c_{t r f} c \dot{\theta}+k_{t l f} z_{u f}-k_{t l f} c \theta+c_{t l f} \dot{z}_{u f}-c_{t l f} c \dot{\theta} \ldots \\
& =k_{t r f} z_{r r f}+c_{t r f} \dot{z}_{r r f}-k_{t r f} \delta_{s t f}+k_{t l f} z_{r l f}+c_{t l f} \dot{z}_{r l f}-k_{t l f} \delta_{s t f}+m_{u f} g+F_{s r f}+F_{s l f}
\end{aligned}
$$

where, $m_{u f}$ is the mass of the front solid axle; $z_{u f}, \dot{z}_{u f}$, and $\ddot{z}_{u f}$ are the displacement, velocity and acceleration of the axle's center of gravity; $k_{t r f}$ and $k_{t f f}$ are the right and left front tire stiffnesses; $c$ is the wheel track half width; $c_{t r f}$ and $c_{t l f}$ are the right and left front tire stiffnesses; $\theta$ and $\dot{\theta}$ are the roll angle and velocity of the front axle; $\delta_{s t}$ is the tire static deflection which is assumed $10 \mathrm{~mm}$ in this study; and $z_{r r f}, \dot{z}_{r r f}, z_{r l f}$ and $\dot{z}_{r l f}$ are the profile heights and their time rates of change on the right and left front axle.

In eq. 3 , besides the suspension forces on the left and right hand sides, the axle experiences the gravitational forces due to its own mass $m_{u f} g$; the forces from the road 
input on the tires and the static forces due to tire deflection $\delta_{s t f}$. Similarly the force equation of motion on the rear axle is given by,

$$
\begin{aligned}
& m_{u r} \ddot{z}_{u r}+k_{t r r} z_{u r}+k_{t r r} c \phi+c_{t r r} \dot{z}_{u r}+c_{t r r} c \dot{\phi}+k_{t l r} z_{u r}-k_{t l r} c \phi+c_{t l r} \dot{z}_{u r}-c_{t l r} c \dot{\phi} \ldots \\
& =k_{t r r} z_{r r r}+c_{t r r} \dot{z}_{r r r}-k_{t r r} \delta_{s t r}+k_{t l} z_{r l r}+c_{t l r} \dot{z}_{r l r}-k_{t l r} \delta_{s t r}+m_{u r} g+F_{s r r}+F_{s l r}
\end{aligned}
$$

where the quantities are similar to those already defined in eq. 3 but, in this case, they refer to the rear axle.

The moment equations on the sprung mass in the roll and pitch directions are given by eq.5 and eq. 6 respectively.

$$
\begin{aligned}
& I_{X X} \ddot{\alpha}=\left(F_{s l r}+F_{s l f}\right) d-\left(F_{s r r}+F_{s r f}\right) d \\
& I_{Y Y} \ddot{\beta}=\left(F_{s l f}+F_{s r f}\right) b-\left(F_{s r r}+F_{s l r}\right) a
\end{aligned}
$$

where $I_{X X}$ and $I_{Y Y}$ are the moments of inertia of the vehicle body in the roll $(\alpha)$ and pitch $(\beta)$ directions respectively; $d$ is half the distance between left and right front suspension struts; $a$ and $b$ are the distances of the rear and front axles from the vehicle's centre of gravity respectively.

The moment equations on the front and rear axles are given by eq. 7 and eq. 8 respectively.

$$
\begin{aligned}
& I_{X X_{-} a} \ddot{\theta}+c k_{t l f} z_{u f}+c^{2} k_{t l f} \theta-c c_{t l f} \dot{z}_{u f}+c^{2} c_{t l f} \dot{\theta}+c k_{t r f} z_{u f}+c^{2} k_{t r f} \theta+c c_{t r f} \dot{z}_{u f}+c^{2} c_{t r f} \dot{\theta} \ldots \\
& =-c k_{t l f} z_{r l f}-c c_{t l f} \dot{z}_{r l f}+c k_{t l f} \delta_{s t f}+c k_{t r f} z_{r r f}+c c_{t r f} \dot{z}_{r r f}-c k_{t r f} \delta_{s t f}+d\left(F_{s r f}-F_{s l f}\right)
\end{aligned}
$$

$$
\begin{aligned}
& I_{X X_{-} a} \ddot{\phi}-c k_{t l r} z_{u r}+c^{2} k_{t l r} \phi-c c_{t l r} \dot{z}_{u r}+c^{2} c_{t l r} \dot{\phi}+c k_{t r r} z_{u r}+c^{2} k_{t r r} \phi+c c_{t r r} \dot{z}_{u r}+c^{2} c_{t r r} \dot{\phi} \ldots \\
& =-c k_{t l r} z_{r l r}-c c_{t l r} \dot{z}_{r l r}+c k_{t l r} \delta_{s t r}+c k_{t r r} z_{r r r}+c c_{t r r} \dot{z}_{r r r}-c k_{t r r} \delta_{s t r}+d\left(F_{s r r}-F_{s l r}\right)
\end{aligned}
$$

where $I_{X X_{-a}}$ is the mass moment of inertia of each axle. 
The equations may now be rewritten in the matrix form

$$
\mathbf{M} \ddot{\mathbf{z}}+\mathbf{C} \dot{\mathbf{z}}+\mathbf{K z}=\mathbf{f}
$$

where the matrices and vectors in eq. 8 are given in References [6, 7].

Eq. 9 is a stiff differential equation owing to the existence of the nonlinear suspension forces and the resulting nature of its stiffness and damping matrices. Hence it is solved by using the low-order routine ode23s.m implemented in MATLAB, which is suited to such stiff differential equations [12]. The standard ODE solver ode $45 . \mathrm{m}$ in MATLAB encounters convergence problems especially in the presence of higher damping, friction and bushing forces. This fact was also noted by Breytenbach [6].

\section{Nominal road profiles}

In the previous study [2], half-sine waves were used to represent road bumps and random road profiles were generated in a purely mathematical way using the wellknown one-dimensional random profile function [2, 13]. In this study, two kinds of road profiles are used: trapezoidal-shaped bump profiles and Belgian paving. The unique feature is that these profiles are based on real measured road surfaces $[8,9]$. The bump profiles were made of trapezoidally shaped steel blocks whose dimensions could be obtained using a one meter rule. The distances along the road were measured using measuring tapes.

The measurements over the Belgian paving were more complicated and therefore required a better profilometer. The profile measures were taken by Becker [8, 9] using three different methods: 'can-can' machine, photogrammetry and laser scanner, which are shown in his work to be accurately correlated [8, 9]. It is however reported that the 'can-can' machine provided a better profile measuring tool due to its ease in setting up, operation and data pre-processing. The 'can-can machine' is a light weight, right-angled triangular structure with a wheel at each of its corners, designed in such a way that one 
wheel is in front and is used for steering the apparatus and controlling its speed during profiling, whereas the other two wheels are at the rear carrying the measuring beam. The rear beam is $4.5 \mathrm{~m}$ long and it carries 30 pivoting arms positioned and spaced to cover a $3 \mathrm{~m}$ wide road section. It is reported that when a 'can-can' machine is properly used it is capable of achieving profiling errors of less than a few millimetres [8, 9]. This accuracy is very satisfactory for the purposes of this study where small bumps may be neglected due to the tire filtering effect $[8,9]$. The vehicle tires tend to cushion the vehicle from the impacts of surface undulations whose wavelengths are less than its contact patch width. A detailed description of the profiling procedure and the equipment used can be found in Becker's work [8, 9].

\subsection{Trapezoidal bumps}

The trapezoidal bumps are used here to represent discrete-obstacle type of defects such as potholes, bumps and stones [6]. Though the real-life discrete obstacles on the road may not often be in that shape, trapezoidal bumps are popularly used in vehicle model validation tests $[8,9,14]$ due to their ramp-type of rise and fall thereby impacting mild harshness to the vehicle structure in driving. Two trapezoidal bumps of different sizes (small bump with $100 \mathrm{~mm}$ height and large bump having a height of $150 \mathrm{~mm}$ ) were placed along the tracks in three different layouts as shown in Figure 2.

The bump layouts are shown in the picture in Figure 2 (a) with the Land Rover traversing the bumps. The spacing between the bumps is depicted in Figure 2(b) to (d). The first layout had two smaller trapezoidal bumps placed symmetrically along each wheel track. This was ideal for testing the vehicle's vertical motion. The second layout, Figure 2 (c) had the large bumps placed symmetrically along each wheel track followed by the small bumps similarly laid out and positioned at a distance of $10.4 \mathrm{~m}$ from the large bumps in the direction of travel. This layout was ideal for testing of both vertical 
and pitch motions. The third layout Figure 2 (d) comprised one large bump on the left hand side of the wheel track followed by the small bump at a distance of $10.4 \mathrm{~m}$ placed on the right hand side of the wheel track. This layout was ideal for testing vehicle's vertical, pitch and roll motions.

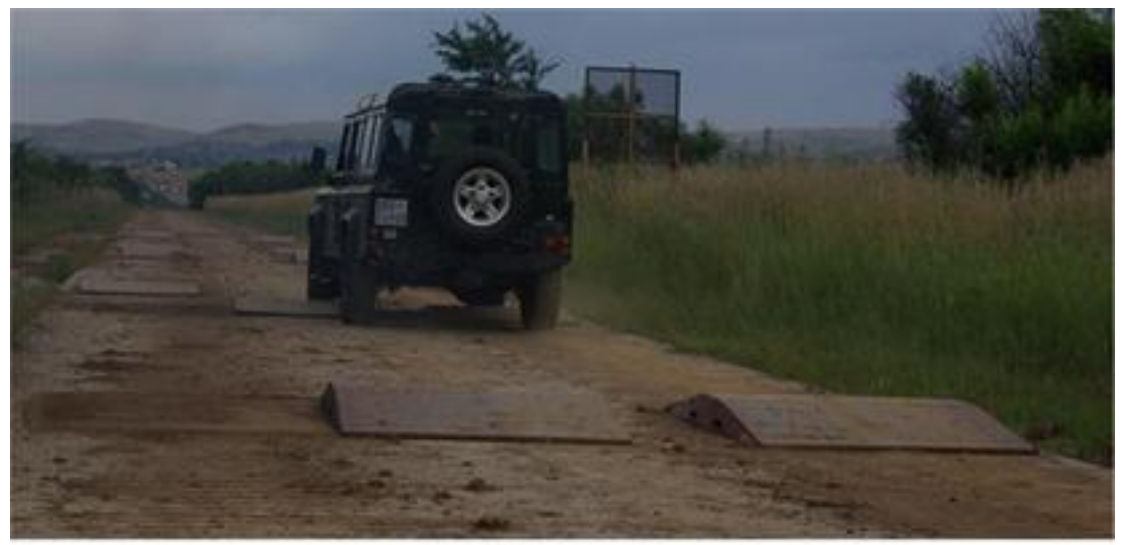

(a)

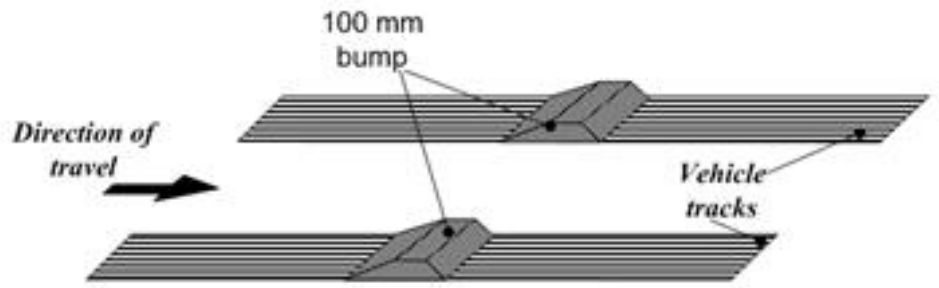

(b)

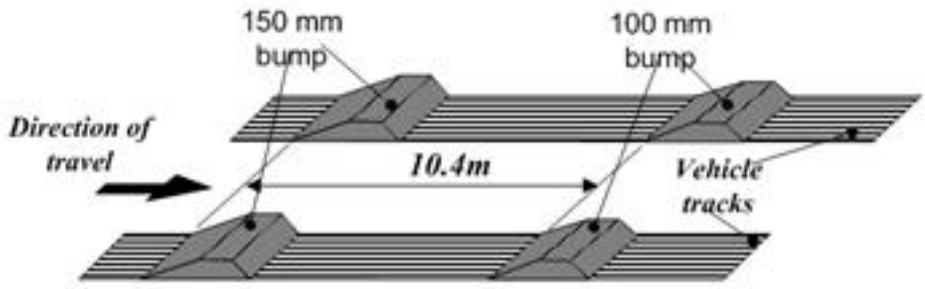

(c)

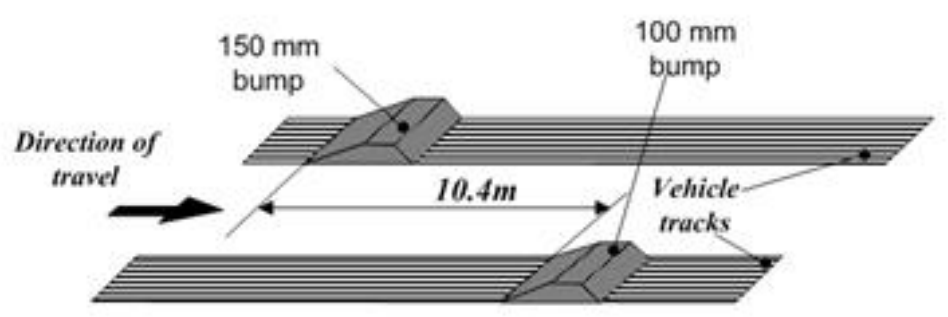

(d)

Figure 2. Bump layouts in picture (a) and in schematic form showing the distances in: (b) symmetric small bump (c) symmetric large-then-small bump (d) asymmetric large-then-small bump. 


\subsection{Belgian paving}

The Belgian paving is often used to evaluate vehicle dynamics typically experienced when traversing random rough terrains $[8,9,14]$. Its surface is made up of cobbles which are firmly cemented together in the pavement. Figure 3 shows the Belgian paving in picture (a), its displacement spectral density (DSD) in (b) and the Land Rover traversing its stretch in (c) at Gerotek. Becker [8, 9] noted that the peak at a spatial frequency of 6 cycles/meter in frame (b) corresponds to a wavelength of $167 \mathrm{~mm}$ which is the average length of the cobbles (a) in the direction of vehicle travel.

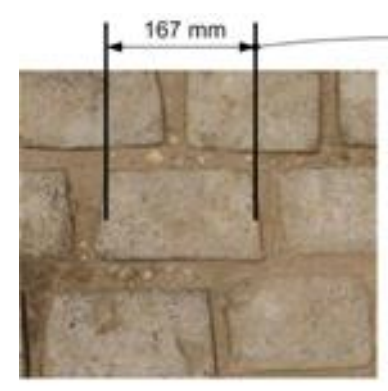

(a)

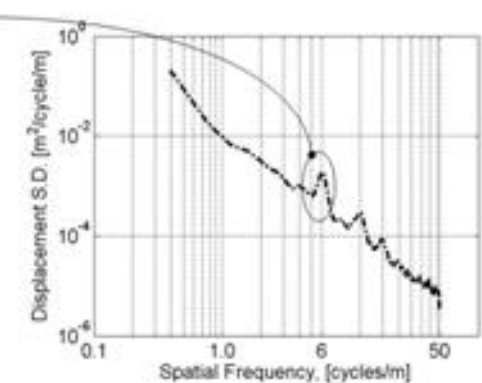

(b)

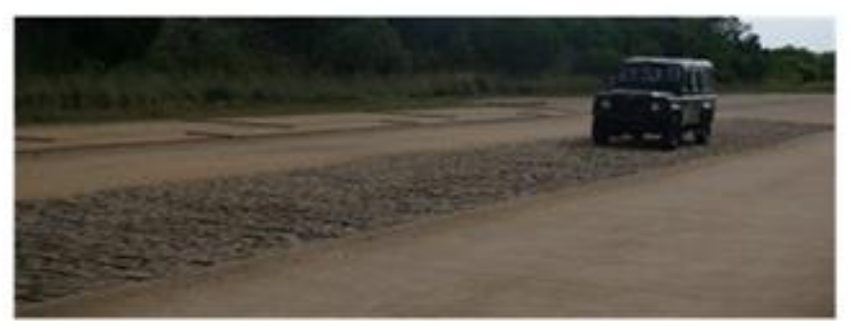

(c)

Figure 3. Belgian paving (a) in picture (b) Displacement Spectral Density plot (c) vehicle on the Belgian paving.

\section{Generating the training data}

The training data is generated by applying slightly adjusted road profiles to the numerical model of eq. 9 in section 2.4. Ideally, the training data should be as representative as possible in order to achieve good network generalization over the testing domain. In this study, the network is to be tested on two geometrically different 
road surfaces and for computational expediency the alterations are applied to these two profile.

For the bumps, the geometrical shapes remain trapezoidal, while the heights (h) and average lengths (x) shown in Figure 4 are resized by a factor of 0.1 and the spacing between the bumps as shown in layouts (c) and (d) shown in Figure 2 are reduced from $10.4 \mathrm{~m}$ to $8 \mathrm{~m}$. Thus the dimensions used in the training algorithm are $(x \pm 0.1 x) \mathrm{m}$ and $(h \pm 0.1 h) m$ for the average widths and heights respectively. This only yielded

geometrically similar trapezoidal bumps with equal corresponding angles, which of course rather simplified the test for network's generalization capabilities over different bump profiles. It is however recommended that in order to achieve better network generalization capabilities over different geometries of bumps, it would be necessary to alter the geometrical shapes and their internal angles besides merely rescaling the heights and widths of the bump blocks.

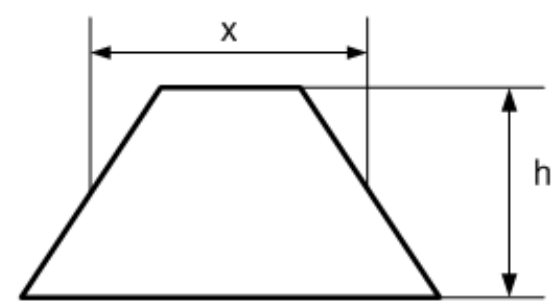

\section{Figure 4. Trapezoidal bump showing height (h) and average length (x).}

For the Belgian paving, a Gaussian noise with a noise-signal ratio of 0.1 and a 1-m delay are added to the spatial profile. The RMS of the Belgian profile is calculated and a noise level equivalent to $10 \%$ of the original spatial profiles' RMS is added to the Belgian paving.

These altered road profiles are applied to the vehicle model under the case scenarios tabulated in Table 2. For each case scenario in Table 2, a matrix consisting of 
accelerations calculated on the front right, rear left and rear right sides of the vehicle body are stored. The accelerations are calculated on corresponding positions of the model to those where accelerometers were positioned on the actual Land Rover. Due to some technical difficulty during the experiments, the accelerations on the left front could not be measured. The stored accelerations are processed to remove any constant trends and low-pass filtered with a cut-off frequency of $25 \mathrm{~Hz}$ to cover the important frequency range for vehicle ride vibrations. The accelerations (inputs) with their corresponding road profiles (targets) provide the training data for the neural network.

Table 2 Summary of road profiles and conditions for generating training data

\begin{tabular}{|c|c|c|c|}
\hline $\begin{array}{l}\text { Case } \\
\text { Scenarios }\end{array}$ & Profile type & $\begin{array}{l}\text { Vehicle } \\
\text { suspension mode }\end{array}$ & $\begin{array}{l}\text { Vehicle speed } \\
(\mathbf{k m} / \mathbf{h})\end{array}$ \\
\hline 1 & Symmetric small bump & Handling & 14.5 \\
\hline 2 & Symmetric large-then-small bump & Handling & 14.5 \\
\hline 3 & $\begin{array}{l}\text { Asymmetric large-then-small } \\
\text { bump }\end{array}$ & Ride & 14.5 \\
\hline 4 & $\begin{array}{l}\text { Asymmetric large-then-small } \\
\text { bump }\end{array}$ & Handling & 14.5 \\
\hline 5 & Belgian paving & Ride & 14.5 \\
\hline 6 & Belgian paving & Handling & 14.5 \\
\hline 7 & Belgian paving & Ride & 54 \\
\hline 8 & Belgian paving & Handling & 54 \\
\hline
\end{tabular}


As shown in the Table 2, there are four case scenarios each under discrete obstacles and under the Belgian paving. It was possible to increase the number of cases for the discrete obstacles according to combinations of suspension settings and vehicle speeds, but that has been intentionally avoided in this study to reduce the resulting computational cost during network training and simulation. In cases of unavailability of a priori knowledge of the test demands, it might be necessary to include as many conditions as possible when generating the training data.

However, the use of such simulated data implied that the performance of the neural network in this paper is largely influenced by the accuracy of the numerical model itself and the quality of the training process. If the training process does not induce much larger errors it might be easier to make some inferences on how the physical parameters of the vehicle system itself impact the final results from the neural network otherwise such inferences may remain hidden and hard to fathom.

The next section presents the neural network and its training function.

\section{ANN architecture and training}

A feed-forward neural network with 50 tan-sigmoid neurons in the hidden layer and two linear neurons in the output layer is used. The network is trained with simulated data from the vehicle model for each of the eight case scenarios presented in Table 2 . The architecture of the static feed-forward neural network employed in this study is shown in Figure 5. This is a departure from the dynamic layer-recurrent NARX used in the previous paper [2]. The reason for the change is that the NARX network requires feedforward from true road profiles which unfortunately may not be available in real test situations. 


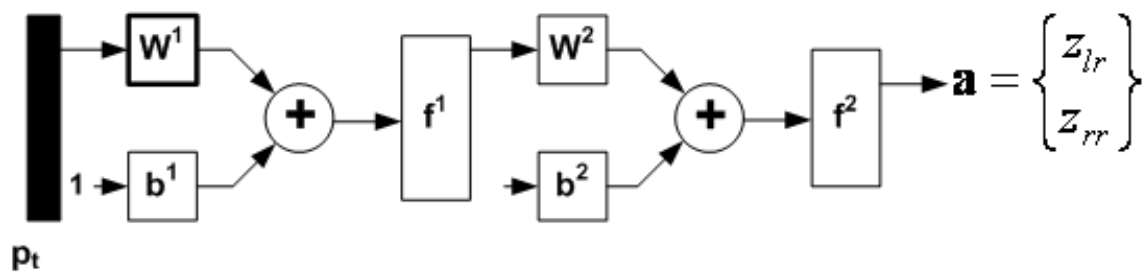

\section{Figure 5. Static feed-forward ANN architecture.}

The input $\mathbf{p}_{t}$ contains three sets of sprung mass accelerations with 3964 data points. This two-layer neural network can be mathematically represented by [12, 15]

$\mathbf{a}=\mathbf{f}^{2}\left(\mathbf{W}^{2} \mathbf{f}^{1}\left(\mathbf{W}^{1} \mathbf{p}_{t}+\mathbf{b}^{1}\right)+\mathbf{b}^{2}\right)$

where $\mathbf{p}_{\mathbf{t}}=\left[\begin{array}{lll}\ddot{\mathbf{z}}_{s f f}(x) & \ddot{\mathbf{z}}_{s l r}(x) & \ddot{\mathbf{z}}_{s r r}(x)\end{array}\right]^{T}$ is a matrix of sprung mass simulated accelerations for the training process on right front, left rear and right rear sides of the vehicle body; $\mathbf{W}^{\mathbf{1}}$ and $\mathbf{b}^{\mathbf{1}}$ are a $50 \times 3$ weighting matrix and a 50-element column bias vector respectively; $\mathbf{f}^{\mathbf{1}}$ comprises 50 sigmoid activation function (tansig.m), in the hidden layer [12] where tansig $(x)=2 /(1+\exp (-2 x))-1$; while $\mathbf{W}^{2}$ and $\mathbf{b}^{2}$ are $2 \times 50$ weight matrix and a 2-element column bias vector respectively being acted on by 2 linear activation functions operating in parallel in $\mathbf{f}^{2}$. The activation in MATLAB function, $\mathbf{f}^{2}$ is implemented by function purelin.m [12]: purelin $(x)=x$. The network output $\mathbf{a}=\left[\begin{array}{ll}z_{l} & z_{r}\end{array}\right]^{T}$ represent the road profiles on the left and right wheel tracks respectively. If $\mathbf{f}^{2}$ is dropped the network output is represented by eq. 11 so that the network output may be rewritten as

$\mathbf{a}=\mathbf{W}^{2} \mathbf{f}^{1}\left(\mathbf{W}^{1} \mathbf{p}_{t}+\mathbf{b}^{1}\right)+\mathbf{b}^{2}$

Roll velocities are not used despite their importance as reported by Breytenbach [6] due to the fact that the numerical model's accuracy in predicting roll velocities is not good. However the application of accelerations from both sides of the vehicle is deemed 
satisfactory to capture the roll effect. The Land Rover had accelerometers instrumented on the vehicle body only at the rear and above the right front suspension strut. This may impose a limitation to the performance of the technique since a position on the chassis does not experience as much excitation as that on the axle. However, in the previous study [2] where all data were generated from the numerical model it was relatively easy to use axle accelerations, and achieve excellent matches between simulated and actual profiles. This presents a further challenge to the application of the methodology in the present set up and, of course, in the real test situation.

In summary, there are a number of important issues to be noted when evaluating the performance of the methodology in the present application against its former application in previous study [2]:

1. A static feed-forward neural network with 50 neurons in the hidden layer and two neurons in the output layer is employed as opposed to the dynamic recurrent NARX network that was employed in the previous study. This is because the NARX network required a feed-forward of true road profiles which are not available in the current application.

2. The network is trained with simulated data generated from altered versions of the real measured road profiles and later tested on measured data. This is done to avoid using the same data in both training and testing the neural network; hence providing a real test to the methodology.

3. The acceleration data used in this investigation are calculated (training data) and measured (testing data) on the vehicle body and not on the vehicle axle. This might provide a limitation on the quality of correlation between simulated and actual road profiles due to the suspension's isolation characteristic. 
4. The network is trained with data at two different speeds and two different suspension settings.

5. The neural network uses the Levenberg-Marquardt algorithm as its learning function. This requires that weights and biases are continuous functions.

6. The accuracy of the calculated accelerations which are used for training the network is limited by the numerical model's deficiencies in predicting roll velocities.

\section{Response measurements}

All the vehicle tests were conducted by Breytenbach [6, 7] on Gerotek's suspension track for wheeled vehicles. These tracks are used to perform repeatable and comparative suspension tests under simulated conditions in order to monitor the structural integrity of body structure, body mountings, suspension, axles, steering, chassis and driveline. Besides, the tests assist in determining and monitoring specific properties, e.g. suspension, steering and structural characteristics.

An eDAQ-lite data acquisition system was used as a data logger. The Crossbow $\pm 4 \mathrm{~g}$ triaxial accelerometers with model number CXL04GP3, were used because of their good response definition at low frequencies, given that important vehicle ride dynamics fall below a frequency of $25 \mathrm{~Hz}$. A sampling frequency of $1 \mathrm{kHz}$ was used with a linear rolloff anti-aliasing filter set at $333 \mathrm{~Hz}$. In the test, several quantities were measured, but for the purpose of this study, only the vertical accelerations and vehicle speeds are of interest. Table 3 shows the parameters of interest. The vehicle speeds were measured by three different methods to ensure repeatability. Right front accelerometers were located on the vehicle strut mount $120 \mathrm{~mm}$ away from the right front axle centre line, while the rear accelerometers were mounted $620 \mathrm{~mm}$ away from the rear axle centre line. It was practically difficult to mount the accelerometers directly on axles near wheel centres. 
Some of the surfaces on which the vehicle was tested were very rough and mounting the accelerometers directly on the axles without a firmer attachnment would compromise the accelerometers themselves. On the other hand, the firmer attachments would necessitate that the accelerometer mounting blocks be bolted into the axle members which would also compromise its structural integrity. But if conditions permit, it is strongly recommended that the accelerations be measured on the vehicle axles.

Table 3 Summary of measured quantities on the Land Rover

Parameter

Time

Vehicle speed

Left rear (LR) vertical acceleration

Right rear (RR) vertical acceleration

Right front (RF) vertical acceleration

\section{Transducer}

eDAQ-lite built-in

probe measuring drive shaft speed

Crossbow triaxial-accelerometer $( \pm 4 \mathrm{~g})$

(CXL04GP3)

Crossbow triaxial-accelerometer $( \pm 4 \mathrm{~g})$

(CXL04GP3)

Crossbow triaxial-accelerometer $( \pm 4 \mathrm{~g})$

(CXL04GP3)

The tests were carried out in random order to reduce the effects of systematic errors in the measurements [6]. The Land Rover was carefully maintained at a constant speed by driving the diesel powered Land Rover against the engine governor in gear [6].

Breytenbach [6] further showed that the tests were repeatable for tests conducted under each case scenario of Table 2. Thus, for testing the neural network in this study, it is satisfactory to select only one test run for each test case. The test data are presented in Table 4. 
Table 4 Summary of test data

\section{Test Case Test Description Suspension Setting Speed $(\mathbf{k m} / \mathbf{h})$}

No. Scenario

\begin{tabular}{|c|c|c|c|c|}
\hline 27 & 1 & $\begin{array}{l}\text { Bump course, } \\
\text { layout (a) }\end{array}$ & Handling & 14.5 (low range, 1 st gear) \\
\hline 22 & 2 & $\begin{array}{l}\text { Bump course, } \\
\text { layout (b) }\end{array}$ & Ride comfort & 14.5 (low range, 1 st gear) \\
\hline 33 & 3 & $\begin{array}{l}\text { Bump course, } \\
\text { layout (c) }\end{array}$ & Ride comfort & 14.5 (low range, 1 st gear) \\
\hline 34 & 4 & $\begin{array}{l}\text { Bump course, } \\
\text { layout (c) }\end{array}$ & Handling & 14.5 (low range, 1 st gear) \\
\hline 10 & 5 & Belgian paving & Ride comfort & 14.5 (low range, 1 st gear) \\
\hline 12 & 6 & Belgian paving & Handling & 14.5 (low range, 1 st gear) \\
\hline 11 & 7 & Belgian paving & Ride comfort & 54 (low range, 4th gear) \\
\hline 13 & 8 & Belgian paving & Handling & 54 (low range, 4th gear) \\
\hline
\end{tabular}

Each test in Table 4 belongs to a particular case scenario presented in Table 2. It can be noted that no data was available for the higher speed on the bump course, due to the severity of the test bump at $54 \mathrm{~km} / \mathrm{h}$.

\section{Simulations, results and discussions}

This section discusses the performance of the network on the real test data. The use of model simulated data for training the network have significant advantages during research and development where models are often used to enhance understanding of underlying complex physical phenomenon and/or optimize system behaviour and 
performance. In the present case however, simulated data have been used to avoid the problem of having to use same data for training and testing or validating the neural network. In sections 6.1 and 6.2 the performance of the network over discrete obstacles and Belgian paving respectively, is presented.

\subsection{Discrete obstacles}

The discrete obstacles are evaluated according to the performance of the methodology over the four bump case scenarios (Table 2 and Table 4), namely: symmetric small bumps in handling mode at vehicle speed of $14.5 \mathrm{~km} / \mathrm{h}$; symmetric large-then-small bumps in ride comfort mode at $14.5 \mathrm{~km} / \mathrm{h}$; asymmetric large-then-small bumps in ride comfort mode at $14.5 \mathrm{~km} / \mathrm{h}$; and asymmetric large-then-small bumps in handling mode at $14.5 \mathrm{~km} / \mathrm{h}$. The bump tests were all carried out at low vehicle speed, to avoid the excessive tire dynamics that may accompany such speeds upon traversing a hard bump such as the one under study. There was also the difficulty of maintaining the vehicle speed when traversing the bump.

For the symmetric small bump, no data is available for the vehicle suspension in ride comfort mode. It has been mentioned previously, that the second bump test scenario provides an ideal situation for testing the vehicle's pitch behaviour. This is true if the distance between the front and rear bumps does not coincide with the vehicle's wheelbase. The third and fourth bump test scenarios excite the vehicle's roll motion and it was carried out for both ride comfort and handling modes. This scenario provides the toughest test in this study since the numerical model does not accurately simulate the vehicle roll dynamics due to its inability to accurately capture the friction in the suspension [6].

The actual bump geometries and layouts are given in Figure 2. The symmetric small bump layout is shown in Figure 2(b) and has a height of $0.1 \mathrm{~m}$. The neural network is 
simulated with the accelerations measured in Test No. 27 in Table 4 to observe its outputs. The reconstructed profiles are shown in Figure 6(a) and (b). The solid lines represent the actual profiles while the dashed lines show the reconstructed profiles. The neural network correctly reconstructs the bumps up to a height of about $0.08 \mathrm{~m}$ after which the simulated profiles exhibit some waviness. The waviness also occurs around the base where the bumps start and end. This network behaviour at and around corners can be expected due to the difficulties associated with numerical integration at and in the neighbourhood of such discontinuities. The cause for the differences in the quality of the reconstructed bump between the left wheel track in Figure 6(a) and right wheel track in Figure 6(b) is not clear at this stage. The minor attenuation in the amplitudes may have been caused by the tire enveloping effect that is not properly taken into consideration in simulation model by the point-follower tire model as well as the averaging effect of the numerical filter applied to the simulated profile. Besides, the point-follower tire model with a parallel linear spring and damper does not accurately represent the tire spring and its non-linear effect wheel hop as well as changes in tire pressure, thereby contributing to some error on the steady-state part of the discrete bumps. Furthermore, this tire model does not accurately simulate the change in tire/ground contact point. However, these shortcomings are tire model-dependent which can be improved if more comprehensive models were used. 

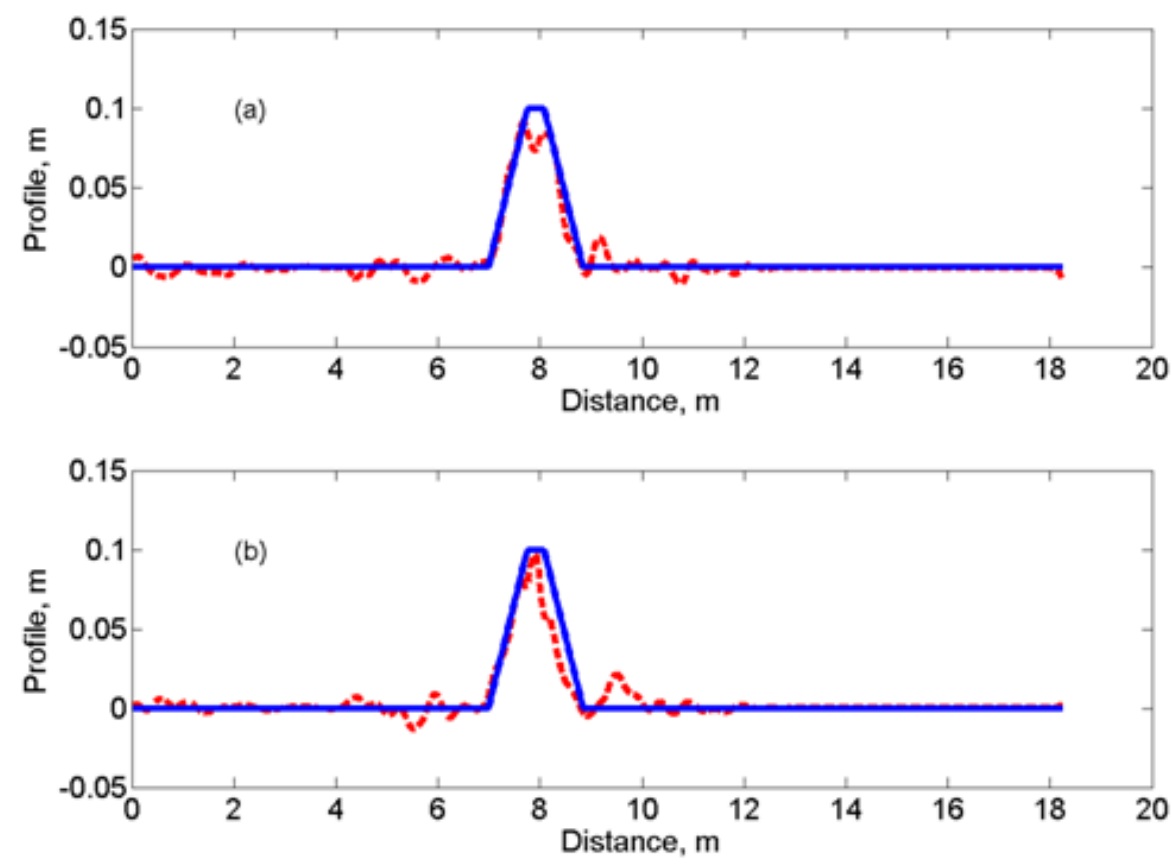

Figure 6. Simulated bumps (dashed) correlated with actual bumps (solid) on the left hand track (a) and right hand track (b) for Case Scenario 1 for Test No 27 in Table 4 (Handling mode at $14.5 \mathrm{~km} / \mathrm{h}$ ).

Figure 7 shows the results of the network simulations over symmetric large-and-thensmall bumps at the vehicle speed of $14.5 \mathrm{~km} / \mathrm{h}$ when the vehicle suspension was set to ride comfort mode as in Case Scenario 2 Test No. 22 in Table 4. The left hand wheel track is represented by Figure 7(a) and the right hand wheel track is shown in Figure 7(b). The reconstructed bumps correlate very closely with the actual profiles on both sides of the wheel tracks. Moderate undulations occur at the corners especially at the bases of the bumps. However, the quality of the reconstructed profiles in terms of heights and general curve fitting is much superior to the Case Scenario 1. Breytenbach [6] noted that the larger number of uncertainties associated with the harder suspension setting in handling mode, affected more adversely the quality of the correlation between simulated and actual vehicle responses than in the more compliant ride comfort mode. 

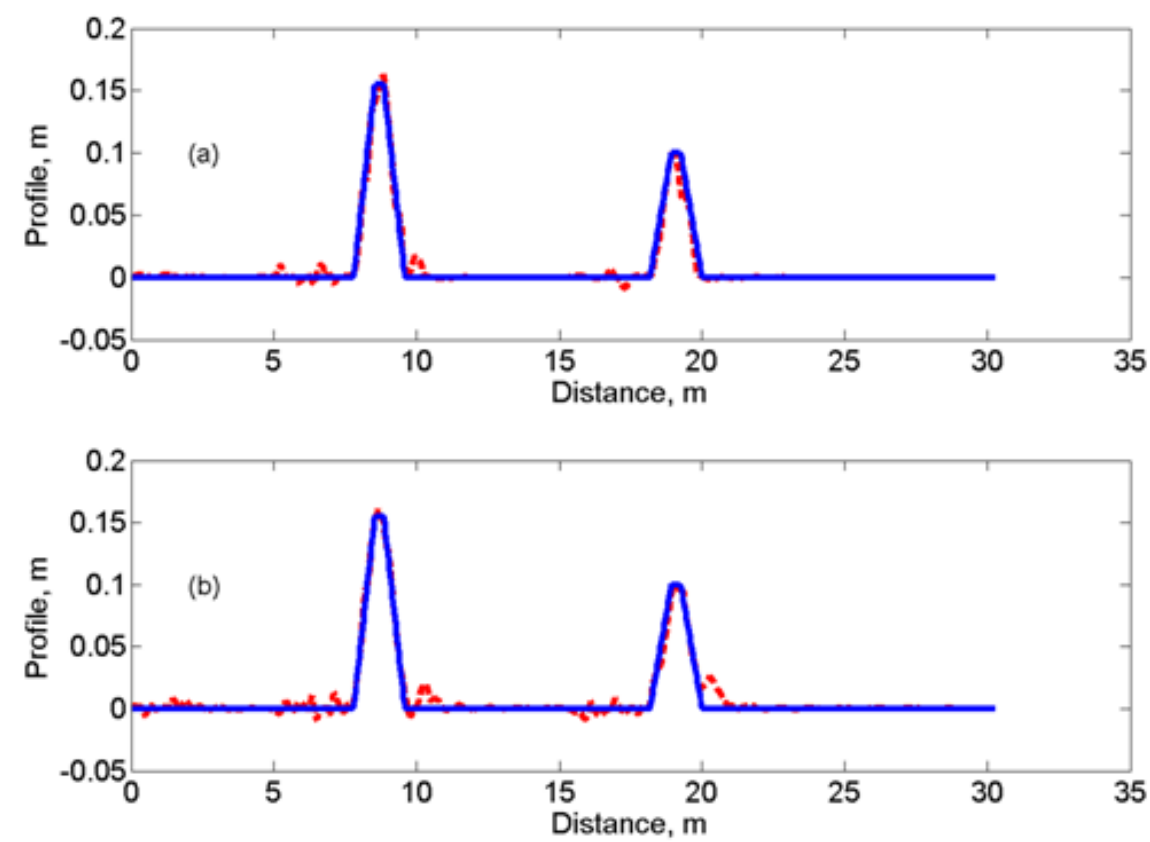

Figure 7. Simulated bumps (dashed) correlated with actual bumps (solid) on the left hand track (a) and right hand track (b) for Case Scenario 2 Test No. 22 in Table 4 (Ride comfort mode at $14.5 \mathrm{~km} / \mathrm{h}$ ).

The performance of the network over asymmetric large-and-then-small bumps are presented in Figure 8 for Case Scenario 3 and in Figure 9 for Case Scenario 4. The reconstructed bumps on the left hand wheel track exhibit better correlation than on the right hand wheel track for both test cases. Though this has been observed in the previous test cases, it is clearly more magnified in these two cases by the fact that the underlying numerical model fails to model roll motion with sufficient accuracy as to allow its attendant neural network to reproduce any form of motion with acceptable level of accuracy, in which roll plays a significant part. Breytenbach noted that the model over-predicted roll velocity and investigated the causes of this error through sensitivity analyses. It was concluded that roll stiffness in suspension bushings, which was not considered in the model, might be culpable for the over-prediction of roll velocities. Frame flexibility was also considered to be a contributor but not necessarily 
the main cause. It is, however, not clear at this stage why these effects seem to affect the ride comfort mode case in Figure 8 more than handling mode case in Figure 9, especially on the right hand wheel tracks. The reconstructed bump in ride comfort mode (Figure 8(a)) does not suffer from a similar energy spill over at the location corresponding to the location of the right hand bump as is observed in Figure 9(a) for handling mode. This might be due to relatively more accurate modelling in the ride comfort mode as compared to more demanding handling mode.
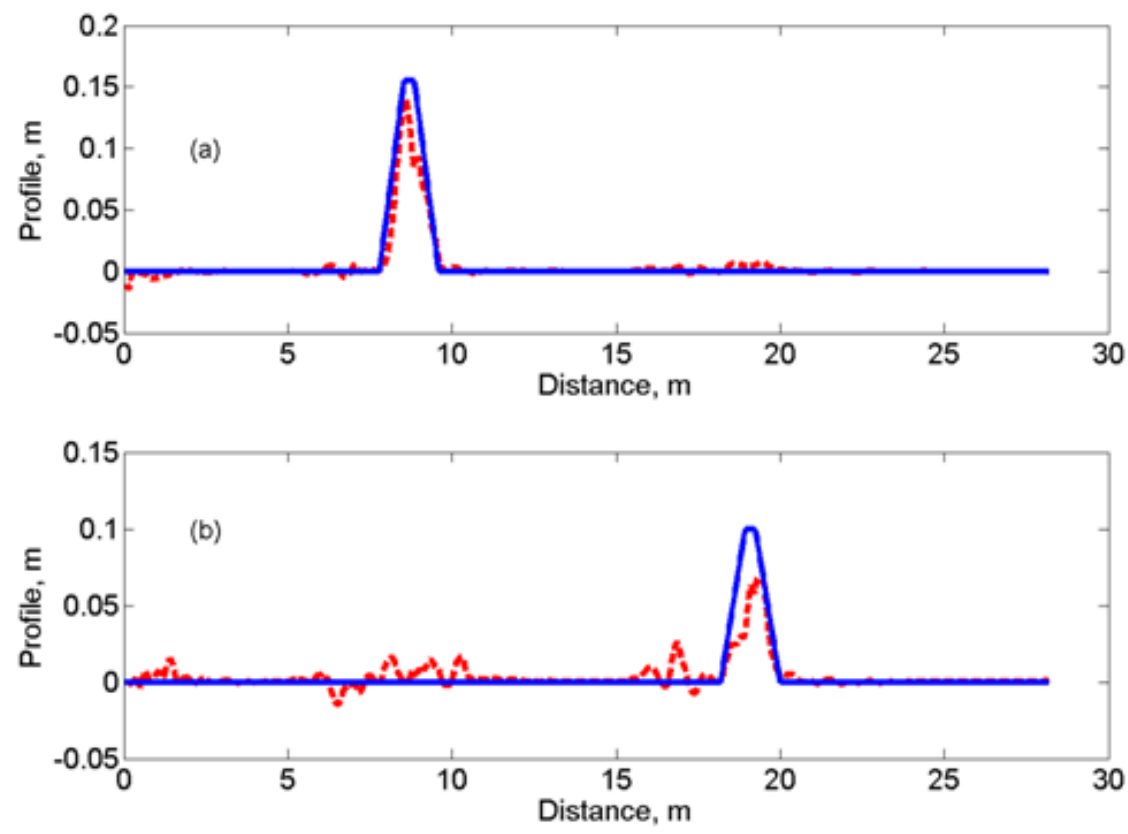

Figure 8 Simulated bumps (dashed) correlated with actual bumps (solid) on the left hand track (a) and right hand track (b) for Case Scenario 3 Test No. 33 in Table 4 (Ride comfort mode at $14.5 \mathrm{~km} / \mathrm{h}$ ). 

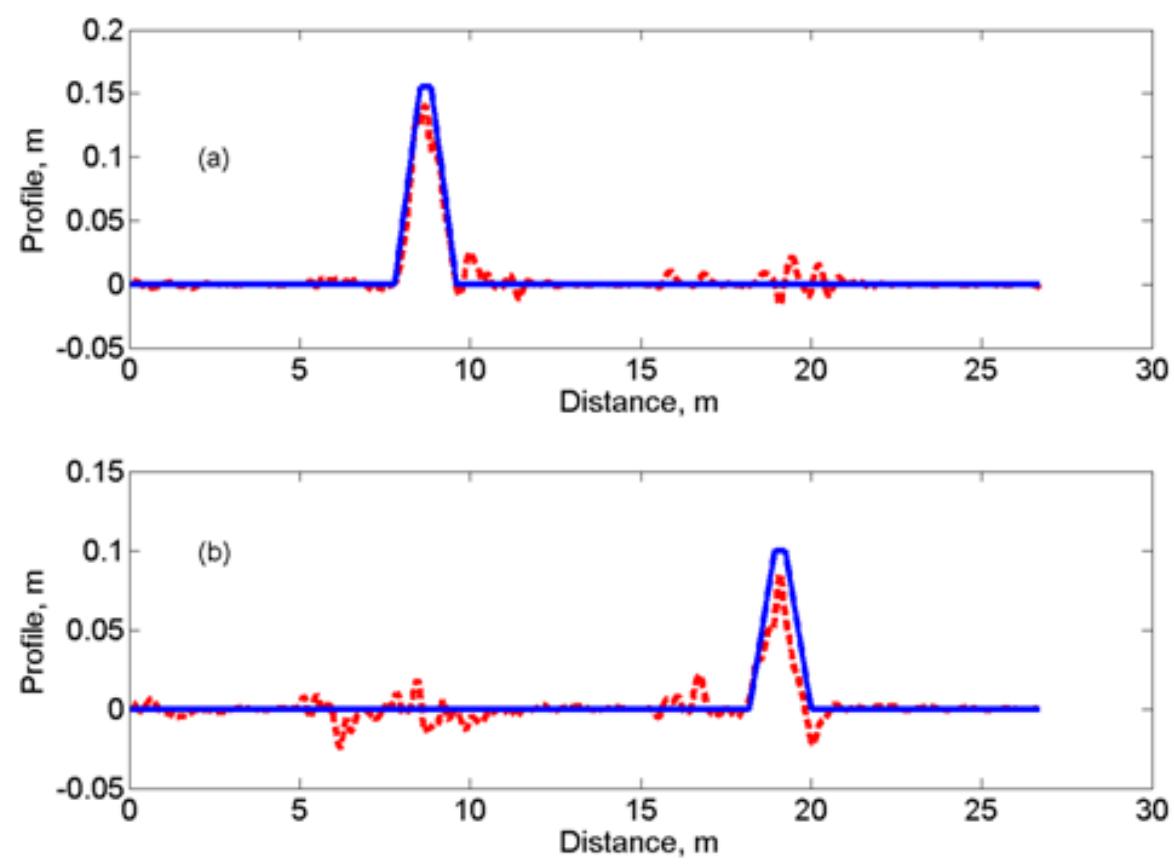

Figure 9. Simulated bumps (dashed) correlated with actual bumps (solid) on the left hand track (a) and right hand track (b) for Case Scenario 4 Test No. 34 in Table 4 (Handling mode at $14.5 \mathrm{~km} / \mathrm{h}$ ).

In this section the performance of the network over different layouts of the discrete obstacles have been presented and discussed. It is observed that the quality of the correlation between the reconstructed and actual bumps is generally better in ride comfort mode than in handling mode. This is a property that is inherited by the neural network from the numerical model that is used to generate the training data. The causes for the differences in the quality of correlations between the left hand and right hand wheel tracks are not clear. There could be a number of factors that may influence this anomaly including vehicle manoeuvre over the bump, inaccuracies in representation of measurement points and slight suspension strut manufacturing disparities.

There are also some errors observed on the steady-state part of the bumps. This may be due to the tyre being modelled as a point follower model with a parallel linear spring and damper. The non-rolling dynamic tire stiffness and damping properties were 
experimentally determined by using a pendulum type tire tester at $200 \mathrm{kPa}$ inflation pressure for a range of vertical loads over tires up to $1600 \mathrm{~kg}$. As a result, a linearized tire stiffness of $250 \mathrm{kN} / \mathrm{m}$ and linearized damping constant of $1.2 \mathrm{kN} . \mathrm{s} / \mathrm{m}$ were recommended for vertical loads of tires below $700 \mathrm{~kg}$. Thus the tire spring and its nonlinear effect wheel hop plus the changes in tire pressure during the actual test are not properly represented by the model thereby contributing to some error on the steady part on discrete bumps. Also the change in tire/ground contact point is not properly simulated by the model.

However the fact that the methodology is still able to yield very good results in the presence of all these uncertainties makes it more practically feasible. The methodology is able to locate the bumps where they occur and is able to estimate the sizes of bumps with maximum discrepancies as shown in Figures 8(b) and 9(b) that might be acceptable for road condition monitoring. It is expected that if the measured data were to be used for training the network some of these offending factors might be eliminated and the methodology would presumably yield better results.

\subsection{Belgian paving}

In the case of Belgian paving tests, it makes more practical sense to correlate their statistical values and DSDs rather than the raw profiles themselves. Heyns, et al. [16] noted that data scarcity may often render profile estimation difficult and as such they proposed the use of a metric calculated from vehicle accelerations. Specifically, Heyns, et al. [16] developed a technique for cost-effective condition monitoring of mine haul roads based on speed normalised response type road roughness measuring systems (RTRRMS) by using a Bayesian framework. Basically they used a Bayesian framework to extract speed normalised RMS acceleration from measured vehicle vibration data. 
The DSDs are used in this study due to their ability to be interpreted as a measure of road roughness $[4,17]$. It is also relatively easy to translate DSDs into International Roughness Indices (IRIs) which themselves are popular measures of road roughness. A similar approach was employed by Kang, Lee and Goo [18] except that they used the DSDs computed from profilometer measured data which might be quite demanding. The approach proposed in this study takes advantage of the current trends in vehicle information systems where vehicles are increasingly being mounted with sensors for optimization of suspension systems.

Figure 10 shows simulated raw profiles plotted over the actual measured profiles. The plots show a limited road section, for clarity purposes only, since the actual training and testing processes were carried out over the entire $100 \mathrm{~m}$ length.
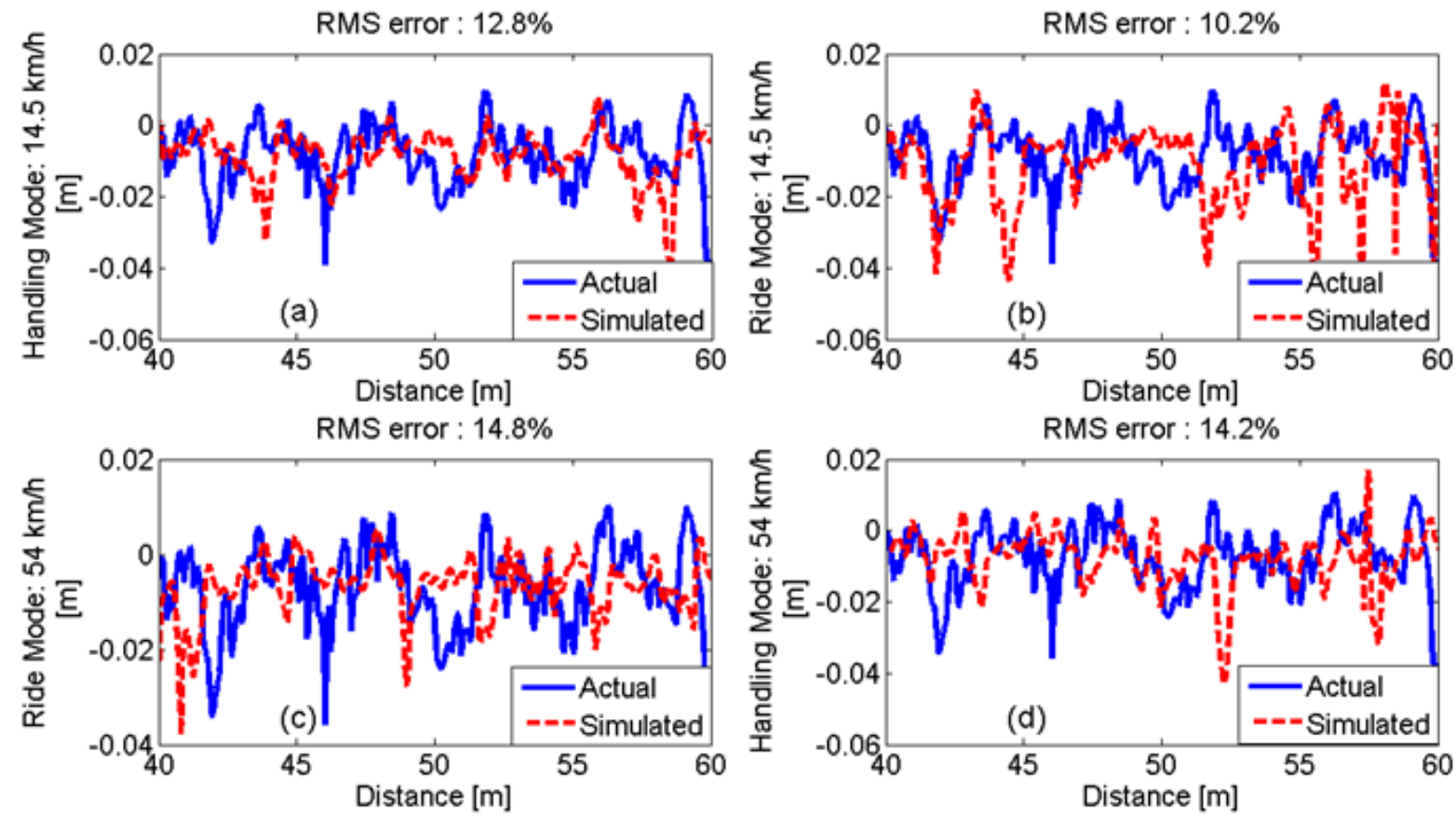

Figure 10. Simulated profiles (dashed) correlated with Actual profiles (solid) for near-optimal and stable training process. 
In this analysis, the RMS error percentage was used to demonstrate correlation between the simulated and the actual road profiles. The RMS error percentages are indicated on top of each plot in Figure 10. The errors are calculated by

$\varepsilon_{r m s}=\frac{\left|y_{r m s}-a_{r m s}\right|}{y_{r m s}} \times 100 \%$

where $y_{r m s}=\sqrt{\sum_{i=1}^{N}\left(y(x)^{2}\right) / N} ; a_{r m s}=\sqrt{\sum_{i=1}^{M}\left(a(x)^{2}\right) / M} ; y(x)$ and $a(x)$ are the measured and simulated profile elevations, respectively, at a distance of $x$ metres along the road; $N$ is the total number of sample points in the measured profile; and $M$ is the total number of sample points in the simulated profile.

The errors in RMS estimations in Figure 10 are below $15 \%$ with ride comfort mode exhibiting both the best correlation of 10.2\% RMS error at the lower vehicle speed of $14.5 \mathrm{~km} / \mathrm{h}$ (Figure $10(\mathrm{~b})$ ), and the worst correlation of $14.8 \%$ RMS error at the higher speed of $54 \mathrm{~km} / \mathrm{h}$ (Figure 10(c)). The performance of the ANN in handling mode lies between these two extremes at $12.8 \%$ for lower vehicle speed (Figure 10(a)) and at 14.2\% for higher vehicle speed (Figure 10(d)). It is observed that the extents of the RMS errors are much higher than those obtained from the numerical model itself, and also, that the relative performance of the ANN model over the Belgian paving did not always agree with the accuracies as obtained from the numerical model. For example there is a ripple effect on the RMS error percentages and the higher vehicle speed does not always yield better correlations than the lower vehicle speed as was the case with the numerical model.

This might be attributed to the rather perverse interplay between the training data and the numerical model itself. The target data for training the ANN was measured from a $100 \mathrm{~m}$ long Belgian paving and that could not be long enough to contain all the 
necessary low frequency information. Furthermore, the numerical model that was used to generate the input data (vehicle body accelerations) had two main deficiencies: first, the implementation of point-follower tire model made the model prone to inducing appreciable levels of higher frequency noise in the calculated accelerations, and secondly, the disregard of wheel-base filtering overestimated spectral energies at some of the frequencies. The combined effects of these two factors negatively affected the stability and convergence abilities of the ANN.

Figure 11 shows the DSDs for the ANN simulated and actual (measured) road profiles plotted over each other for each combination of vehicle suspension mode and speed on a road roughness scale. The results show that the DSDs are very well correlated above a wavenumber of 0.2 cycles $/ \mathrm{m}$ (corresponding to a wavelength of $5 \mathrm{~m}$ ). The DSDs on the lower frequency portion are relatively poor due to insufficient low frequency data content in the $100 \mathrm{~m}$ long road. The roads are consistently classified as D in the midfrequency range between 0.2 to 3.0 cycles $/ \mathrm{m}$ and $\mathrm{C}$ to $\mathrm{A}$ in the higher frequency ranges. The exception exists at and around the wavelengths corresponding to the cobble width of $0.17 \mathrm{~m}$ where the DSDs are amplified. 

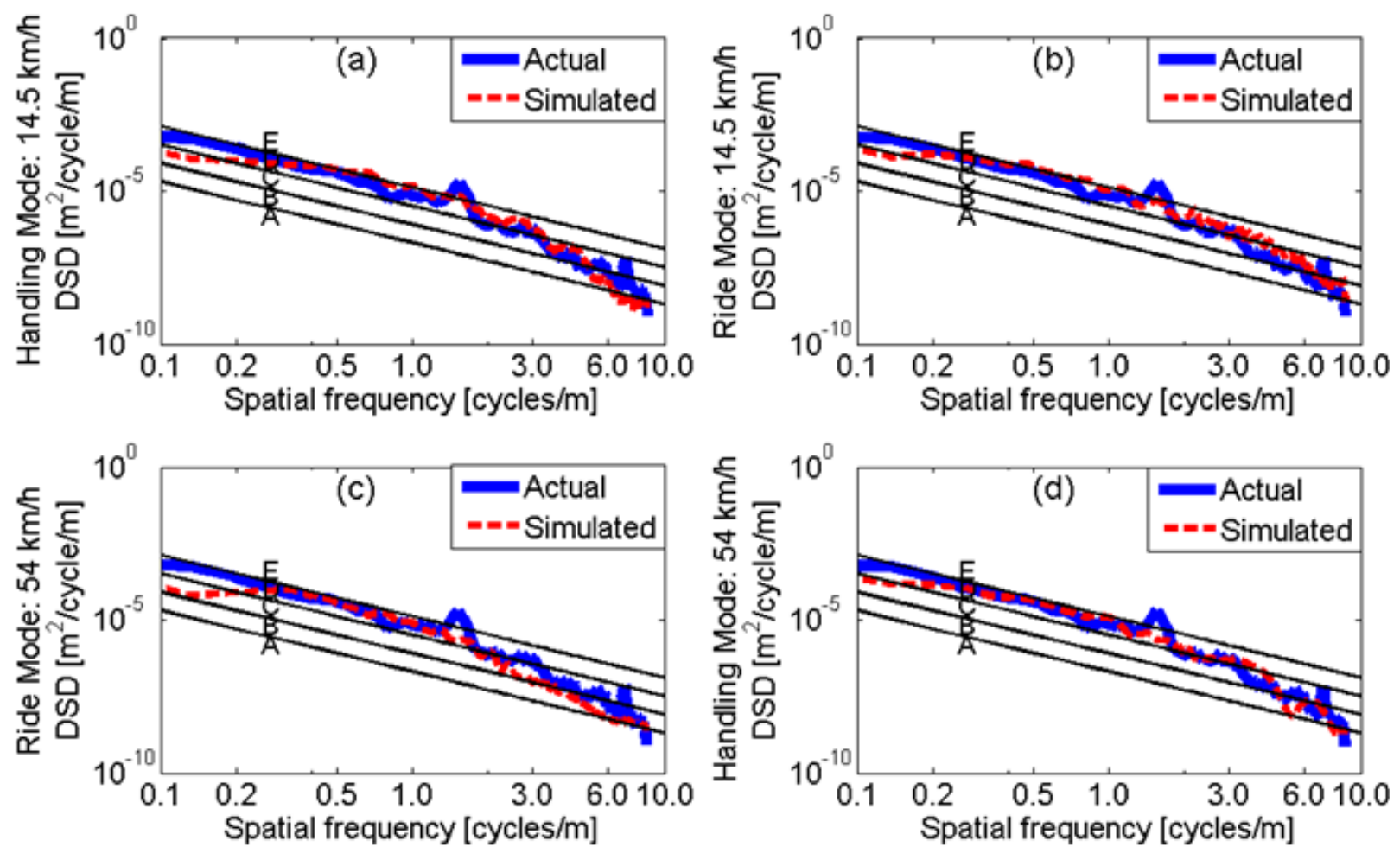

Figure 11. Simulated DSDs (dashed) correlated with Actual DSDs (solid) for near-optimal and stable training process.

The ANN training and simulation processes were carried out on a Dell XPS L502X laptop with Intel Core i7 CPU at 2.2GHz having a random access memory (RAM) of 8GB and running on a 64-bit Windows. It has a hard drive disk (HDD) storage of 750 GB. The total duration in training and simulation of the ANN was measured as $65 \mathrm{~s}$ out of which $2 \mathrm{~s}$ was for simulation. Thus for a pre-trained ANN the short duration in simulation can benefit an online condition monitoring system.

\section{Conclusions}

In this paper a methodology for estimating road profiles and roughness classes has been 
demonstrated by simulating an artificial neural network on measured data. The neural network was trained with data calculated by a numerical model of the Land Rover. This was deemed necessary due to lack of sufficient data to be used for both training and testing the neural network. The training data was obtained by applying altered versions of the real road profiles to the numerical model. This presented an opportunity to test the network with data that were different from the data that was used to train it and hence test its generalization capabilities.

Eight different test cases were drawn from a combination of road profiles, vehicle suspension modes and speeds. The road profiles comprised discrete obstacles, from which three different layouts were constructed, and the Belgian paving. The vehicle suspension was adjusted between ride comfort and handling modes while the vehicle was either driven at $14.5 \mathrm{~km} / \mathrm{h}$ or $54 \mathrm{~km} / \mathrm{h}$. For all bump layouts, the vehicle speed was kept at the lower speed of $14.5 \mathrm{~km} / \mathrm{h}$.

The methodology has been applied differently to the two road profiles without necessarily departing from the central theme of the study of providing a means for road condition monitoring. The ANN reconstructed profiles were correlated with actual measured profiles for both discrete obstacles and the Belgian paving. However in the discrete obstacles, only overlay plots of the simulated and actual bump profiles were found satisfactory, while for the Belgian paving, overlay plots as well as RMS error percentages were used to provide a measure of accuracy in correlation. The overlay plots of discrete obstacles show very good correlations between ANN simulated bumps and actual bumps for all cases under investigation. The results showed superior quality in the reconstructed bumps for ride comfort as compared to handling mode. However, 
the overall network performance over bumps was observed to be marred by the poor roll motion estimation capabilities of the numerical model as well as its use of the pointfollower tire model. This shortcoming shrinks the validity area but it is only modeldependent. If, on the other hand, a more comprehensive vehicle model is used, the validity area is effectively enlarged.

The Belgian paving however presented its own peculiar challenges due to combined effects caused by the deficiencies in the training data and the numerical model that was used to generate the data. Thus ANN training was carried out in the absence of sufficient training data. As a result, three typically different cases were identified from the simulated results (see Appendix A): first case, the ANN could achieve near-optimal state where the RMS error percentages were all below 15\%; second case, the ANN could only manage to converge to an overly sub-optimal state where the RMS error percentages were largely between 15 and $25 \%$; and third case, the ANN could be trapped in an unstable region and the resulting RMS error percentages were largely above $25 \%$.

For the first case (with stable and near-optimal training process), the simulated profiles matched the actual profiles very well and the evaluated RMS error limit of $15 \%$ lies within the error margin of $20 \%$ as per the findings of the previous study[2] by the author. The simulated profile DSDs showed perfect correlation with the actual profile DSDs for all frequencies above 0.2 cycles $/ \mathrm{m}$. In the second case, the ANN would either yield very good correlations over the entire range of frequencies above 0.2 cycles $/ \mathrm{m}$ as in the first case, or it would yield poor estimations of the spectral densities at frequencies higher than 1.8 cycles/m. Besides converging to sub-optimal weights that might yield underestimation of some of the outputs; neural network generalization might have enforced high frequency filtering on the simulated profiles. The third case 
was characterized by spiky spectral densities at the higher frequency end particularly beyond 2 cycles $/ \mathrm{m}$.

However, these two problems are not viewed as absolutely debilitating to the application of the methodology to road condition monitoring. The findings in this study show that the methodology has a lot of potential. Firstly, the methodology is able to locate the bumps where they occur and is able to estimate the sizes of bumps with practically acceptable maximum discrepancies. Secondly, the consistency with which accurate estimations of the DSDs for the Belgian paving, have been made in the frequency range between 0.2 cycles $/ \mathrm{m}$ and 1.8 cycles $/ \mathrm{m}$, for all three cases, is an extremely encouraging result. Though such stable frequency ranges may vary from one vehicle-road test scenario to another, it might yet be useful to identify such frequency ranges for any given specific test scenario so that practically useful road roughness estimations could be made irrespective of the state of the training process. In this study, the errors would have been alleviated if the attendant numerical model was made more accurate by replacing the point follower tire model with a more accurate tire model. Then low-frequency data scarcity due to short Belgian paving would have been dealt with by generating the training data from much longer road lengths $(600-1000 \mathrm{~m})$ that closely resembled the Belgian paving.

\section{Acknowledgements}

The support of the National Research Foundation under the South African Co-operation Fund for Scientific Research and Technological Developments is gratefully appreciated. I also remain greatly indebted to Mr Carl M. Becker of Vehicle Dynamics Group for making available the measured data on the Belgian paving and for the technical advice that he readily offered in the use of the data. 


\section{Appendix A}

It was very hard to consistently converge to the same or approximately similar weights and more so, to a stable region during different training sessions. In order to deal with this problem in this study, several training sessions were run and typical results are plotted in Figures A.1 - A.6. The specific impacts of each vehicle suspension mode are not clear owing to the relatively higher magnitudes of the RMS errors introduced by the training process itself.

The results shown in Figure 10 represent the case of near-optimal performance for the ANN training process. However two more different cases were identified. The first case is where the neural network converged to an overly sub-optimal state where some of the resulting RMS error percentages lay between $15 \%$ and $25 \%$. In the second case the neural network could not achieve any convergence but it would simply be trapped in an unstable region where the resulting weights yielded impractically high output values (RMS error percentages above $25 \%$ ). The overly sub-optimal performance was typified by the results as shown in Figures A.1 - A.4 while Figures A.5 and A.6 represent the unstable performance. 

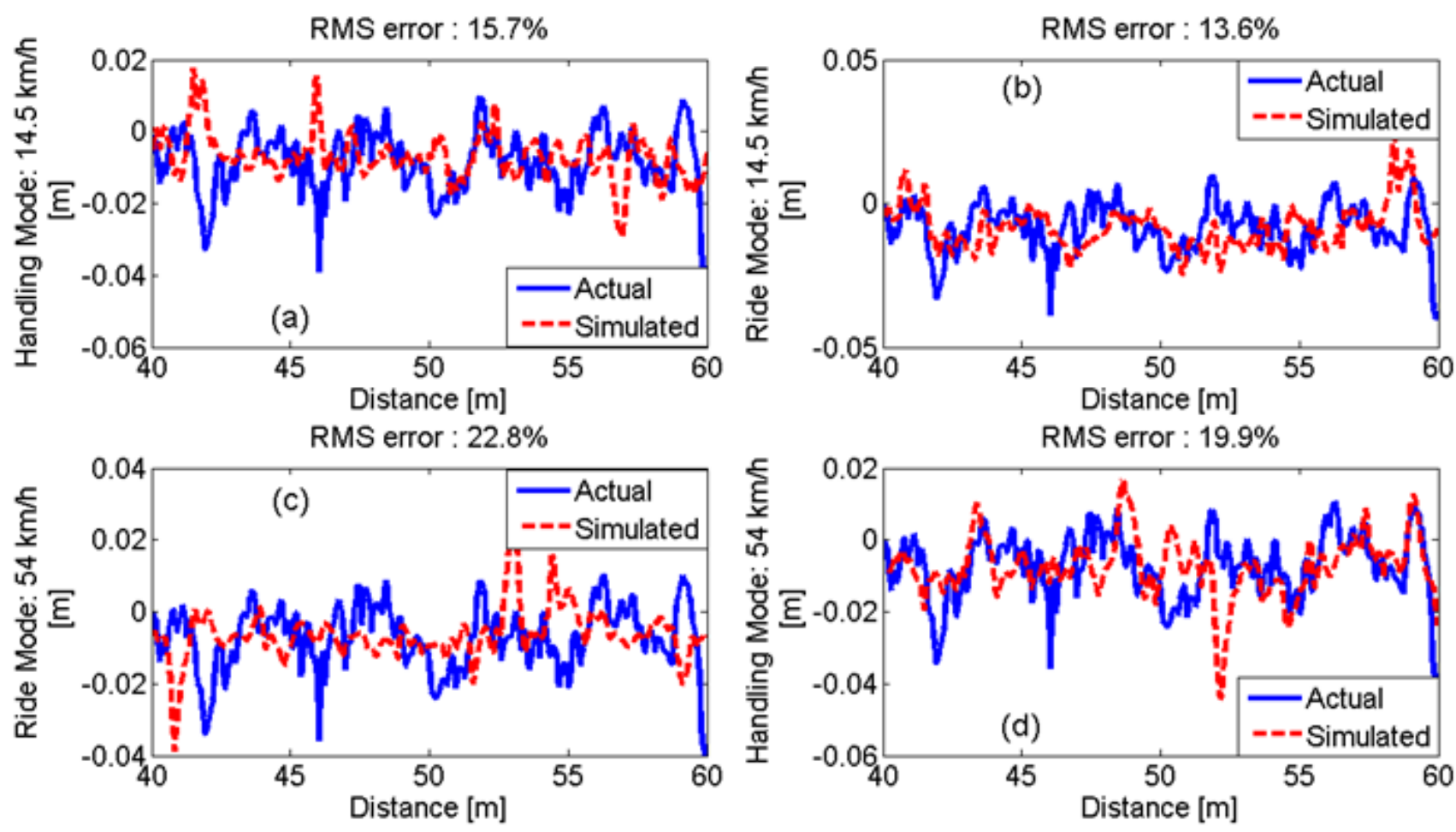

Figure A.1. Simulated profiles (dashed) correlated with Actual profiles (solid) for sub-optimal and stable training process.

Figure A.1 shows results that are similar in nature to Figure 10 except the differences in the magnitude of the RMS error percentages. The simulated profiles are better correlated at lower vehicle speeds (Figures A.1(a) and A.1(b)) than higher vehicle speeds (Figures A.1(c) and A.1(d)) for both vehicle suspension modes. The ANN exhibits much better performance for ride comfort mode (Figure A.1(b)) than handling mode (Figure A.1(a)) at lower vehicle speed while, at higher vehicle speed, better ANN performance was obtained from handling mode (Figure A.1(d)) than ride comfort mode (Figure A.1(c)). 

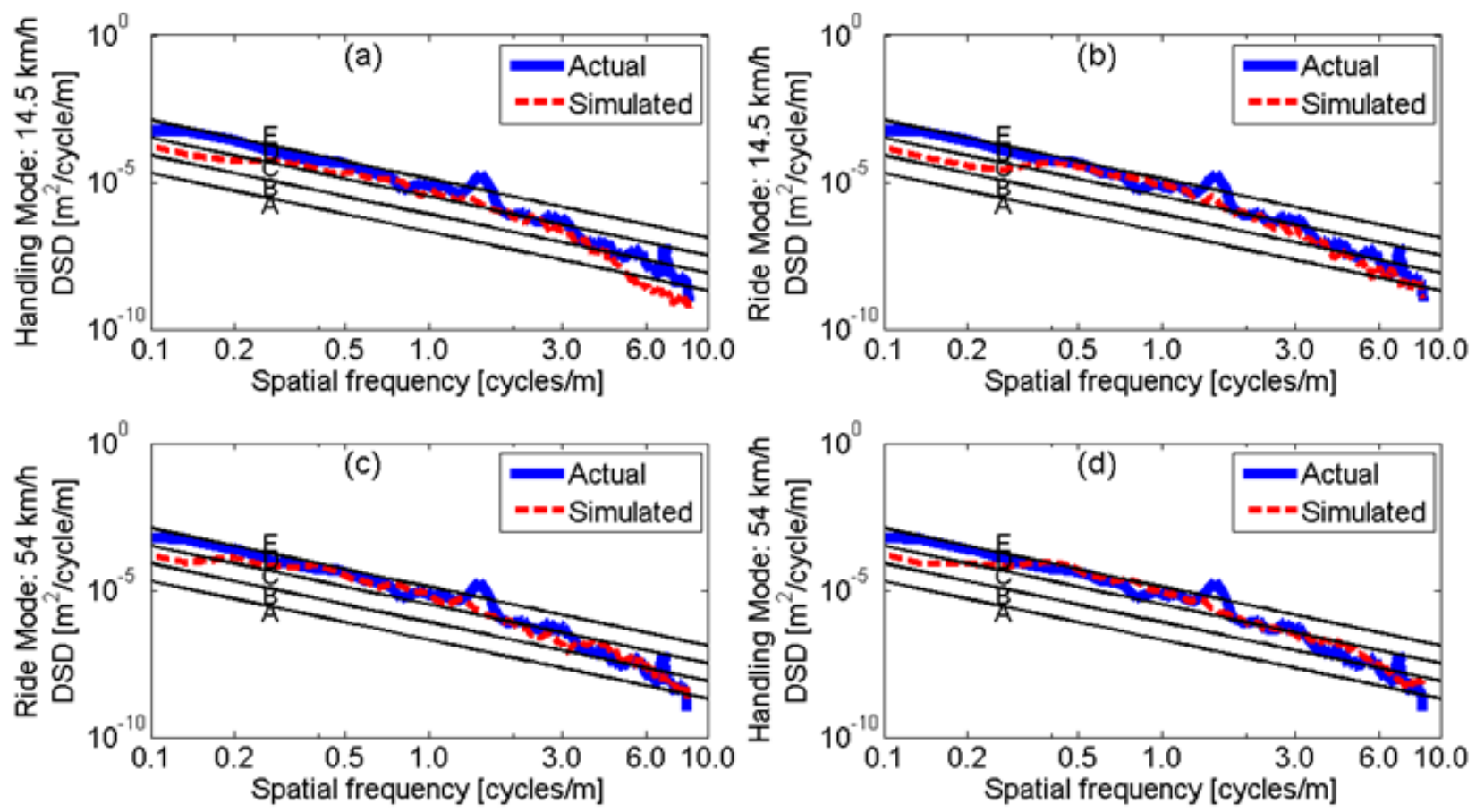

Figure A.2. Simulated DSDs (dashed) correlated with Actual DSDs (solid) for sub-optimal and stable training process.

Despite overly sub-optimal performance in the training process, the DSDs for the simulated profiles in Figure A.2 are very well correlated with the DSDs for actual profiles. There are insignificant differences from the DSDs plotted in Figure 11. Similar low frequency errors as shown in Figure 11 and already discussed in Section 7.2 occur in Figure A.2. This is where the application of ANN and DSD evaluations to road maintenance decision making could be potentially useful. The DSDs are not too sensitive to errors introduced by small amount of sub-optimality in the training process of the ANN. 

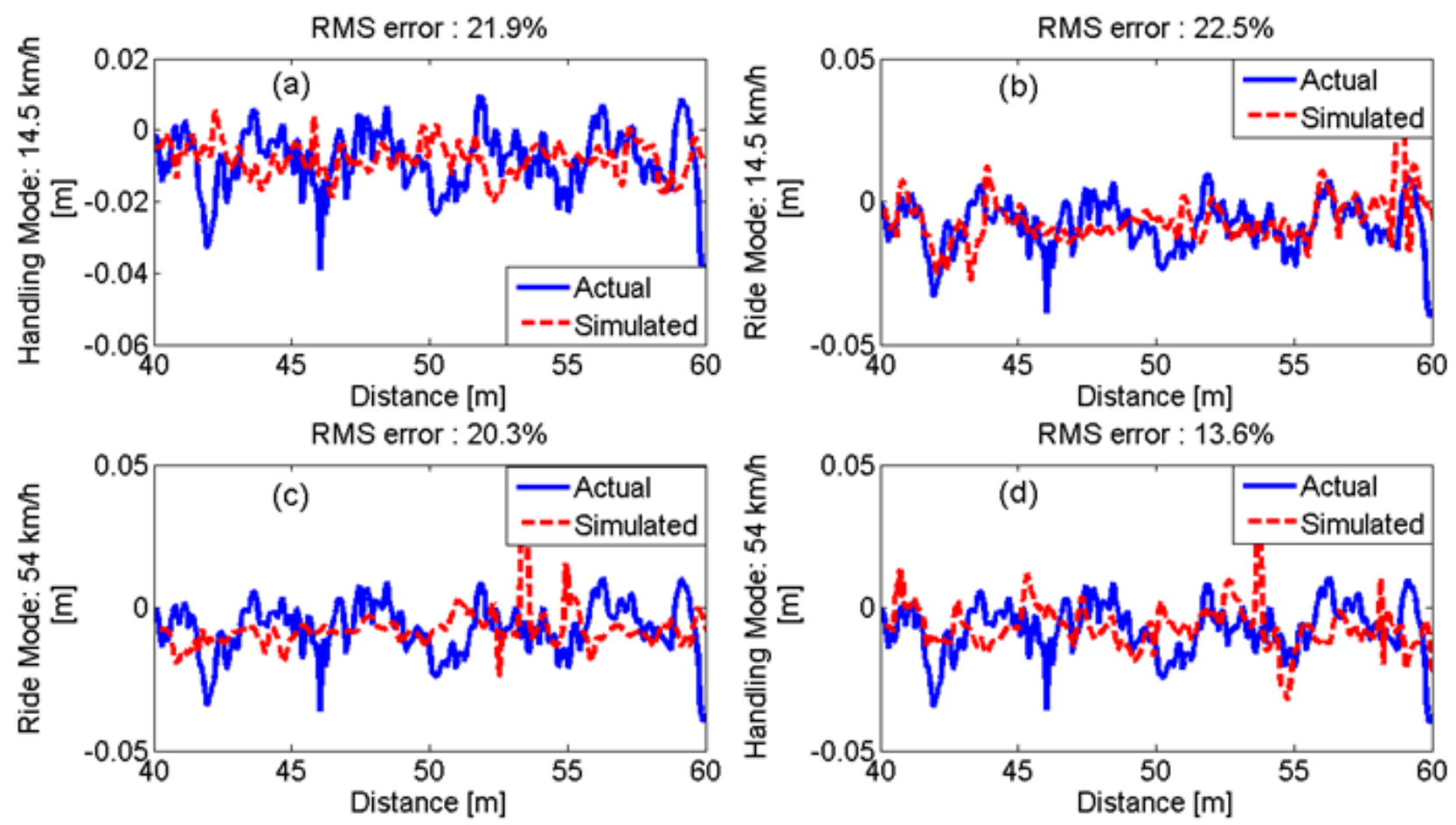

Figure A.3. Simulated profiles (dashed) correlated with Actual profiles (solid) for sub-optimal and stable training process.

Figure A.3 shows different ANN performance from that shown in Figures 10 and A.1.

The neural network produces better correlated results at higher vehicle speed (Figure

A.3(c) and A.3(d)) than at lower vehicle speed (Figure A.3(a) and A.3(b)) and the neural network yields better correlations in handling mode (Figure A.3(a) and A.3 (d)) than ride comfort mode (Figure A.3(b) and A.3(c)) at both vehicle speeds. With the exception of the latter observation, this seems to agree well with the performance of the numerical model itself but as pointed in Section 7.2 it might be hard to fathom specific vehicle suspension influences given the magnitude of error induced by the training process itself. 

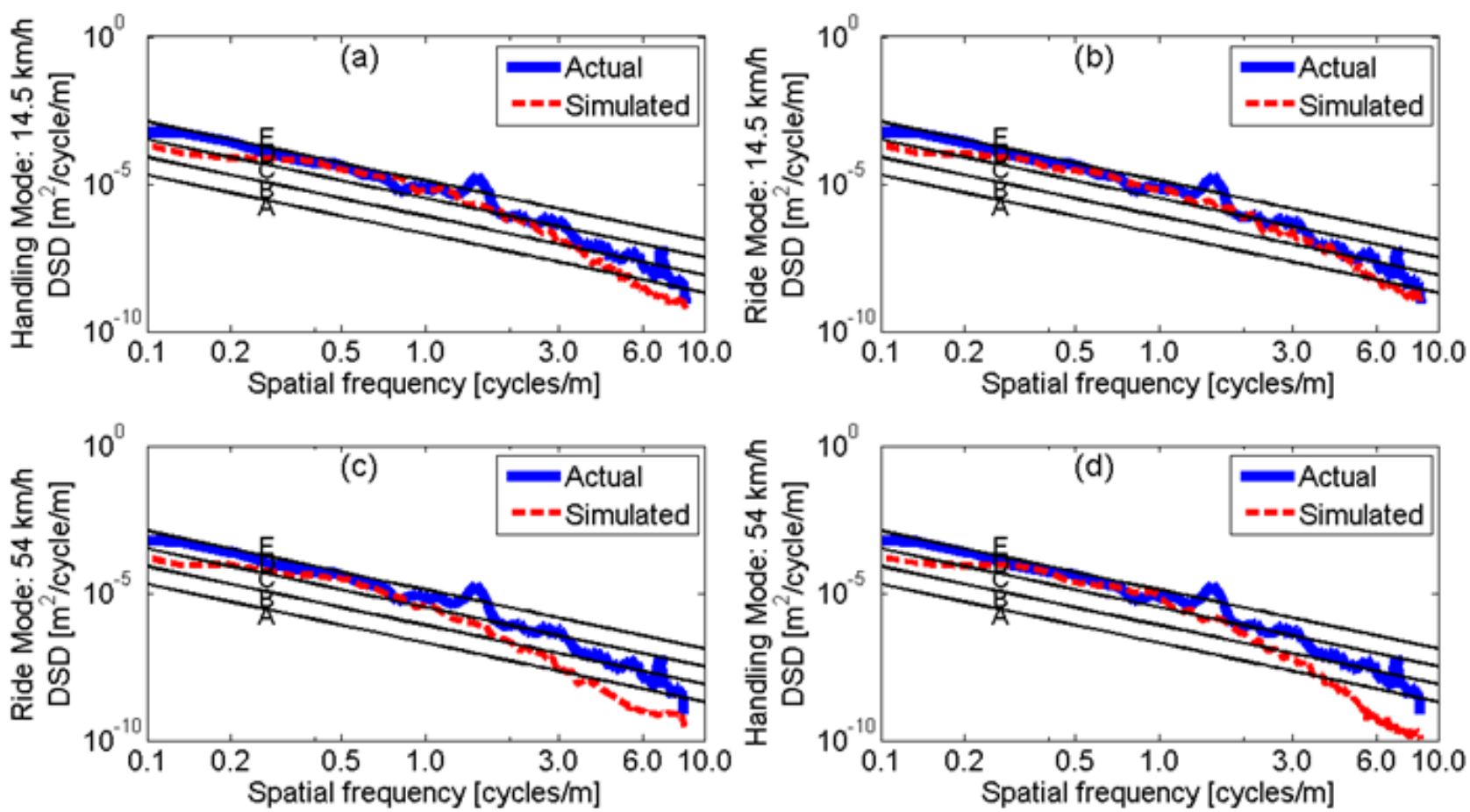

Figure A.4. Simulated DSDs (dashed) correlated with Actual DSDs (solid) for sub-optimal and stable training process.

Figure A.4 exhibits a potential shortcoming of the DSD analysis. Despite the better raw profile correlation at higher vehicle speeds as shown in Figure A.3, the resulting DSDs in Figures A.4(c) and A.4(d) are not as well correlated at higher frequencies (above 1.8 cycles/m). However one can still use the information from mid-frequency range between 0.2 cycles $/ \mathrm{m}$ to 1.8 cycles $/ \mathrm{m}$ and then apply a linear extrapolator to the higher frequencies to estimate their corresponding spectral densities. 

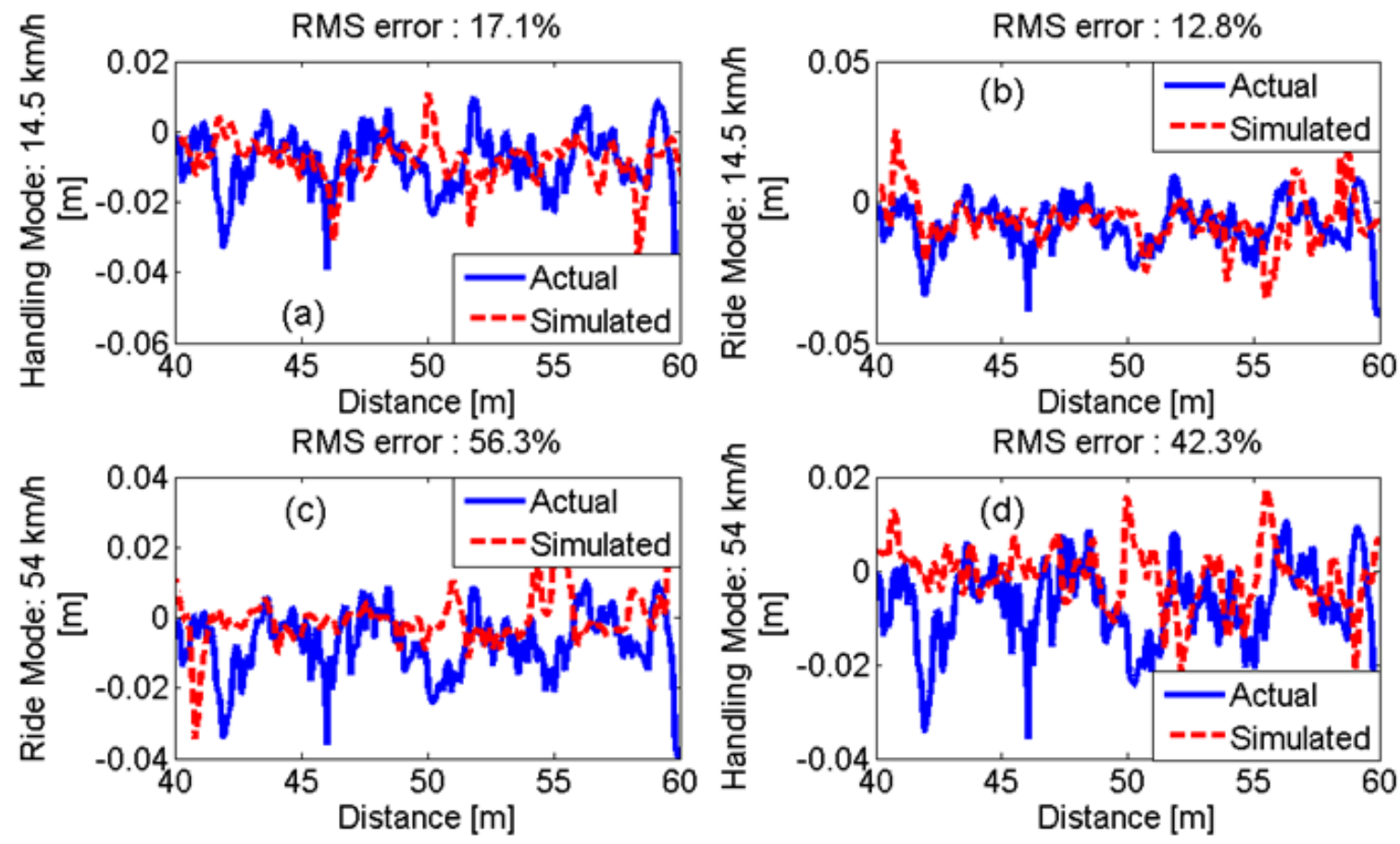

Figure A.5. Simulated profiles (dashed) correlated with Actual profiles (solid) for unstable training process.

Substantial RMS error percentages occurred at higher vehicle speeds for the unstable training process shown in Figure A.5. The ANN performed reasonably well for ride comfort mode at lower speed only (Figure A.5(b)). DSD plots show that the problem affected certain high frequencies (Figures A.6(c) and A.6(d)). The range between 0.2 and 1.8 cycles/m remain unaffected by this problem such that it is also possible to apply the linear extrapolator, as in the previous case, for estimation of the spectral densities at the higher frequencies. 

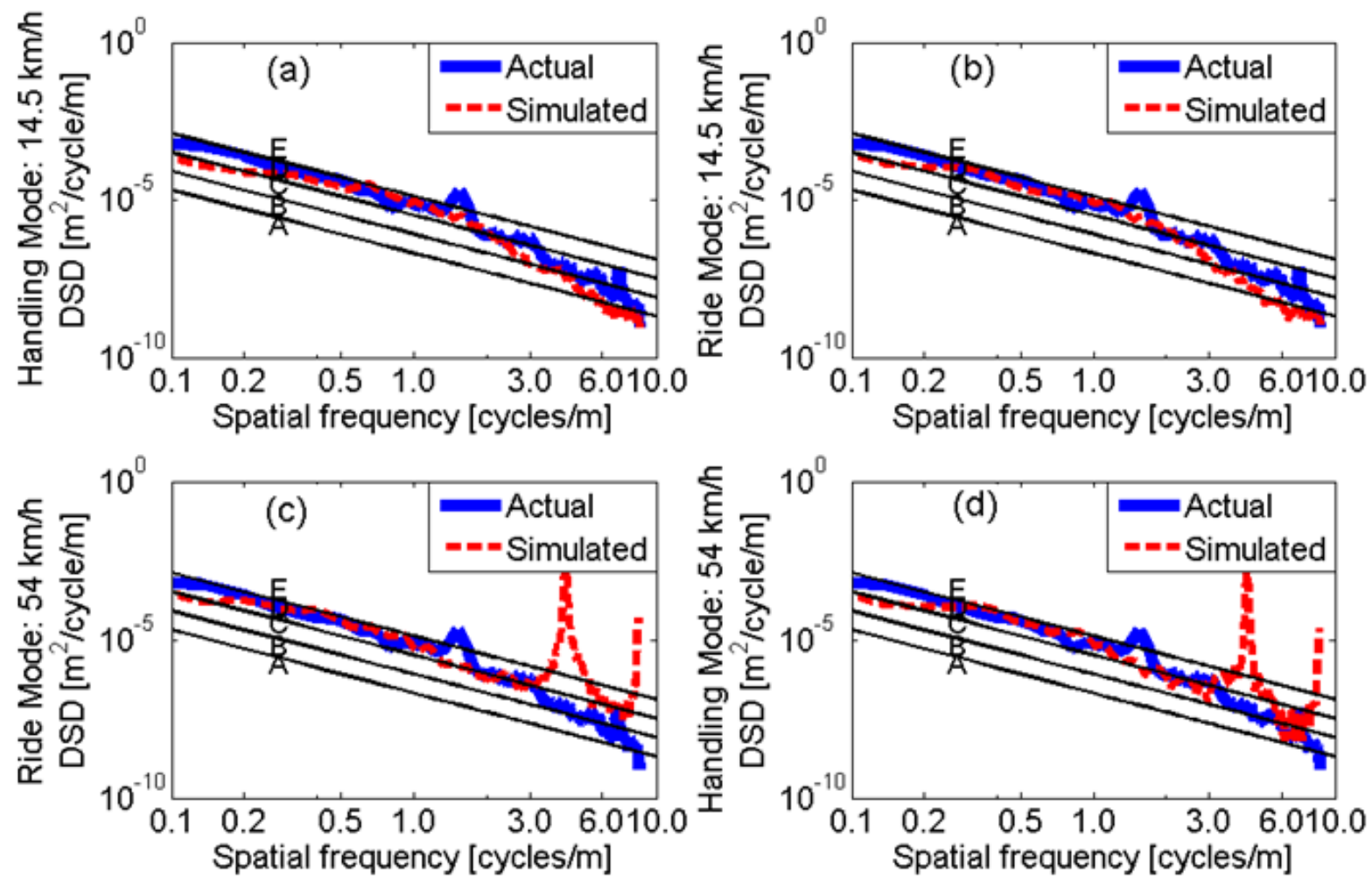

Figure A.6. Simulated DSDs (dashed) correlated with Actual DSDs (solid) for unstable training process.

\section{References}

[1] Hugo D., Heyns P.S., Thompson R.J., Visser A.T. Haul road defect identification and condition assessment using measured truck response, Journal of Terramechanics 2008; $45: 79-88$.

[2] Ngwangwa H.M., Heyns P.S., Labuschagne F.J.J., Kululanga G.K. Reconstruction of road defects and road roughness classification using vehicle responses with artificial neural networks simulation. Journal of Terramechanics 2010; 47:97-111.

[3] Heyns T., De Villiers J.P., Heyns, P.S. Consistent haul road condition monitoring by means of vehicle response normalization with Gaussian processes. Engineering Applications Artificial Intelligence 2012; 25:1752 - 1760. 
[4] International Organization for Standardization ISO 8608: 1995(E), Mechanical vibration - Road surface profiles - Reporting of measured data, 1995.

[5] Els, P.S. The ride comfort vs. handling compromise for off-road vehicle. PhD Thesis, Department of Mechanical and Aeronautical Engineering, University of Pretoria, South Africa, 2006. http://upetd.up.ac.za/thesis/submitted/etd-07152008102911/unrestricted/00front.pdf.

[6] Breytenbach H.G.A. Optimal vehicle suspension characteristics for increased structural fatigue life. MEng Dissertation, Department of Mechanical and Aeronautical Engineering, University of Pretoria, South Africa, 2009.

http://upetd.up.ac.za/thesis/available/etd-09172010-190101/unrestricted/dissertation.pdf

[7] Breytenbach B., Els P.S. Optimal vehicle suspension characteristics for structural fatigue life. Journal of Terramechanics 2011; 48:397-408.

[8] Becker C.M. Profiling rough terrain. MEng Dissertation, Department of Mechanical and Aeronautical Engineering, University of Pretoria, South Africa, 2008. http://upetd.up.ac.za/thesis/available/etd-11262009-171410/unrestricted/00front.pdf

[9] Becker C.M., Els P. Profiling of rough terrain. International Journal of Vehicle Design (Article in Press).

[10] Uys P.E., Els P.S., Thoresson M.J., Voigt K.G., Combrinck W.C.. Experimental determination of moments of inertia for an off-road vehicle in a regular engineering laboratory. International Journal of Mechanical Engineering Education 2006 34(4) 291-314.

[11] Gillespie T.D., Fundamentals of vehicle dynamics. Society of Automotive Engineers, Inc., Warrendale, 1992.

[12] Mathworks Inc., MATLAB Help Tutorial. http://www.mathworks.com/products. 
[13] Cebon D. Handbook of vehicle-road interaction, Swets \& Zeitlinger Publishers, Lisse, 1999.

[14] Letherwood M.D., Gunter D.D. Ground vehicle modelling and simulation of military vehicles using high performance computing, Parallel Computing 2001; 27: 109-140.

[15] Bishop, C.M. Neural networks for pattern recognition, Oxford University Press, Oxford, 1995.

[16] Heyns T., Heyns, P.S., De Villiers J.P. A method for real-time condition monitoring of haul roads based on Bayesian parameter estimation. Journal of Terramechanics 2012; 49:103 - 113.

[17] Andrén P. Power spectral density approximations of longitudinal road profiles. International Journal of Vehicle Design 40 (1, 2, 3) (2006).

[18] Kang D.-K., Lee S.-H., Goo S.-H. Development of standardization and management system for the severity of unpaved test courses. Sensors 7 (2007) 20042027, http://www.mdpi.org/sensors/papers/s7092004.pdf. 